\title{
The most general second-order field equations of bi-scalar-tensor theory in four dimensions
}

\author{
Seiju Ohashi, ${ }^{a}$ Norihiro Tanahashi, ${ }^{b}$ Tsutomu Kobayashi $^{c}$ and Masahide Yamaguchi $^{d}$ \\ ${ }^{a}$ Cosmophys Group, IPNS KEK, \\ 1-1 Oho, Tsukuba 305-0801, Japan \\ ${ }^{b}$ DAMTP, Centre for Mathematical Sciences, University of Cambridge, \\ Wilberforce Road, Cambridge CB3 OWA, U.K. \\ ${ }^{c}$ Department of Physics, Rikkyo University, \\ Toshima, Tokyo 175-8501, Japan \\ ${ }^{d}$ Department of Physics, Tokyo Institute of Technology, \\ Tokyo 152-8551, Japan \\ E-mail: sohashi@post.kek.jp, N.Tanahashi@damtp.cam.ac.uk, \\ tsutomu@rikkyo.ac.jp, gucci@phys.titech.ac.jp
}

ABSTRACT: The Horndeski theory is known as the most general scalar-tensor theory with second-order field equations. In this paper, we explore the bi-scalar extension of the Horndeski theory. Following Horndeski's approach, we determine all the possible terms appearing in the second-order field equations of the bi-scalar-tensor theory. We compare the field equations with those of the generalized multi-Galileons, and confirm that our theory contains new terms that are not included in the latter theory. We also discuss the construction of the Lagrangian leading to our most general field equations.

Keywords: Cosmology of Theories beyond the SM, Classical Theories of Gravity

ARXIV EPRINT: 1505.06029 


\section{Contents}

1 Introduction 1

2 Construction of the most general equations of motion 3

2.1 Assumptions 3

2.2 Second-order rank-2 tensor whose divergence is of second order 4

2.3 Consequence of eq. (2.5) 7

2.4 The most general second-order equation of motion 11

3 Comparison with the generalized multi-Galileon theory 11

4 Candidate Lagrangian $\quad 13$

$\begin{array}{lll}5 & \text { Discussions and summary } & 15\end{array}$

$\begin{array}{ll}\text { A The } \xi \text { tensors } & 16\end{array}$

$\begin{array}{ll}\text { B Explicit form of } \mathcal{Q}_{I} & 16\end{array}$

$\begin{array}{ll}\text { C Euler-Lagrange equations } & 17\end{array}$

$\begin{array}{ll}\text { D Construction of Lagrangian: single scalar-field case } & 18\end{array}$

\section{Introduction}

The inflationary scenario [1-3] is now regarded as a necessary ingredient of modern cosmology because the recent observations of cosmic microwave background anisotropies [4-7] strongly suggest the epoch of inflationary expansion in the early Universe. Since inflation is typically driven by scalar field(s), scalar-tensor theories provide a firm framework to study the dynamics of inflation. On the other hand, the unknown energy called dark energy is shown to be dominant in the present Universe [8-10] and might be understood as an outcome of infra-red modification of gravity. Scalar-tensor theories provide powerful tools to realize such a modification, and their phenomenological features have been studied intensively to confront them against observations. Although a plethora of inflationary models and modified gravity theories have been proposed thus far, unfortunately, we have not yet succeeded in finding the real theory and have kept seeking for it. We have two options to address this problem. The first one is to pursue the ultimate (real) theory on the basis of theoretical consistencies, which is often called top-down approach. The other is to construct a framework of theories as general as possible, which is called bottom-up approach. Of course, though both of approaches are complementary, one of the merits to take the latter approach is to give a unified understanding of various models proposed individually. Another is that one can easily pin down or constrain models once characteristic observational results would be reported. 
The Horndeski theory [11] provides a typical working example of the latter approach because it is the most general single scalar-tensor theory with second-order field equations. It is shown [12] that the Horndeski theory is equivalent to the generalized Galileon [13]. In fact, almost all of the inflationary models with single inflaton proposed so far can be described by this theory in a unified manner. Various aspects of single field inflationary models have been studied in this framework [12], which is useful for us to constrain the models from observational results.

In this paper, we take the latter approach and try to extend the Horndeski theory, which includes only one scalar degree of freedom, to the bi-scalar case. ${ }^{1}$ Although no observational results suggest the presence of multiple (light) scalars during inflation, our framework can clarify the essential difference between single and multiple scalar cases, and is useful for further constraining or detecting the presence of multiple scalar degrees of freedom from observations. Such an attempt to extend the Horndeski theory has already been discussed. First of all, multi-field Galileon theory was proposed in the flat spacetime [19-22]. Later, the covariantization of this multi-field Galileon theory, called generalized multi-Galileon, was considered [23] and conjectured that the theory would correspond to the multi-field extension of the Horndeski theory, that is, the most general multi-field scalar-tensor theory with second order equations of motion. Though the multifield Galileon theory in the flat spacetime is proven to be the most general multiple-scalar field theory in the flat space-time with second order scalar equations of motion [24], it was shown that the generalized multi-Galileon is not the most general theory [25] because this theory does not contain the multi-DBI inflation models [26-30], in particular the doubledual Riemann term appearing in these models. Motivated by these considerations, in this paper, we try to construct a multi-field extension of the Horndeski theory. Especially we focus on the extension to the bi-scalar case as a first step.

The organization of this paper is as follows. In section 2, we construct the field equations of the most general two-scalar tensor theory with second order field equations following Horndeski's procedure. This section is the main part of this paper. In section 3, we compare our theory with the generalized multi-Galileon theory. We show that terms which are missing in generalized multi-Galileon theory are actually contained in our theory. In section 4, we comment on the construction of the Lagrangian corresponding to the field equations we obtain. Finally we summarize our paper and discuss the results in section 5 .

Notations and conventions. Before closing the introduction, we summarize the notations and conventions used throughout this paper. We consider a four-dimensional spacetime with a metric $g_{a b}$ and two scalar fields $\phi^{I}$ with $I=1,2$. Following ref. [11], derivatives of $g_{a b}$ and $\phi^{I}$ with respect to the coordinates $x^{a}$ are denoted as

$$
g_{a b, c} \equiv \frac{\partial g_{a b}}{\partial x^{c}}, \quad \phi_{, a}^{I} \equiv \frac{\partial \phi^{I}}{\partial x^{a}}
$$

\footnotetext{
${ }^{1}$ Another direction is to consider a scalar-vector theory instead of a scalar-tensor theory. Motivated by the earlier work of Horndeski [14], construction of the most general vector theory with second order field equations on flat space, called the vector-Galileon theory, was attempted in ref. [15]. Recently, the scalar-tensor theory with higher order equations of motion without introducing the ghost was proposed as well $[16,17]$. See also [18] for yet another possible way of extending the Horndeski theory.
} 
respectively. We denote the covariant derivative of $\phi^{I}$ with respect to $g_{a b}$ and its scalar product respectively as

$$
\phi_{\mid a}^{I} \equiv \nabla_{a} \phi^{I}, \quad X^{I J} \equiv-\frac{1}{2} \phi_{\mid a}^{I} \phi^{J \mid a},
$$

where $X^{I J}$ is symmetric in $I$ and $J$. We use a strike "| " also as a separator in (anti-)symmetrization. For example, $[I|J K, L| M]$ stands for anti-symmetrization of $I$ and $M$. Partial derivatives of a function $A^{a \ldots b}\left(g, \partial g, \partial^{2} g, \phi^{I}, \partial \phi^{I}, \partial^{2} \phi^{I}\right)$ are expressed as

$$
\begin{aligned}
& A^{a \ldots b ; c d} \equiv \frac{\partial A^{a \ldots b}}{\partial g_{c d}}, \quad A^{a \ldots b ; c d, e} \equiv \frac{\partial A^{a \ldots b}}{\partial g_{c d, e}}, \quad A^{a \ldots b ; c d, e f} \equiv \frac{\partial A^{a \ldots b}}{\partial g_{c d, e f}}, \\
& A_{I}^{a \ldots b ;} \equiv \frac{\partial A^{a \ldots b}}{\partial \phi^{I}}, \quad A_{I}^{a \ldots b ; c} \equiv \frac{\partial A^{a \ldots b}}{\partial \phi_{, c}^{I}}, \quad A_{I}^{a \ldots b ; c d} \equiv \frac{\partial A^{a \ldots b}}{\partial \phi_{, c d}^{I}},
\end{aligned}
$$

and partial derivatives of a function $A\left(\phi^{I}, X^{J K}\right)$ are expressed as

$$
A_{, I} \equiv \frac{\partial A}{\partial \phi^{I}}, \quad A_{, I J} \equiv \frac{1}{2}\left(\frac{\partial A}{\partial X^{I J}}+\frac{\partial A}{\partial X^{J I}}\right)
$$

In the equations of motion and the Lagrangian, we use the generalized Kronecker delta defined by

$$
\delta_{j_{1} \ldots j_{n}}^{i_{1} \ldots i_{n}} \equiv n ! \delta_{\left[j_{1}\right.}^{i_{1}} \ldots \delta_{\left.j_{n}\right]}^{i_{n}}, \quad \delta_{J L}^{I K} \equiv 2 \delta_{[J}^{I} \delta_{L]}^{K}
$$

Repeated indices are summed over $a=0,1,2,3$ and $I=1,2$.

\section{Construction of the most general equations of motion}

The first step of the construction of the most general scalar-tensor theory of ref. [11] is to work out the most general equations of motion that are of second order in derivatives and compatible with the general covariance. In this section, we generalize this construction to the case with two scalar fields.

\subsection{Assumptions}

The assumptions imposed on the theory we are going to construct are summarized as follows.

1. The theory has a Lagrangian scalar density, $\mathcal{L}$.

2. The Lagrangian scalar density, $\mathcal{L}$, is composed of a metric, two scalar fields, and their derivatives up to arbitrary order:

$$
\mathcal{L}=\mathcal{L}\left(g_{a b}, g_{a b, c}, g_{a b, c d}, \ldots ; \phi^{I}, \phi_{, a}^{I}, \phi_{, a b}^{I}, \ldots\right),
$$

where $I=1,2$. 
3. Field equations are composed of the metric, the two scalar fields, and their derivatives up to second order:

$$
\begin{gathered}
0=\frac{\delta \mathcal{L}}{\delta g_{a b}}=\sqrt{-g} \mathcal{G}^{a b}\left(g_{c d}, g_{c d, e}, g_{c d, e f} ; \phi^{J}, \phi_{, c}^{J}, \phi_{, c d}^{J}\right), \\
0=\frac{\delta \mathcal{L}}{\delta \phi^{I}}=\sqrt{-g} \mathcal{E}_{I}\left(g_{a b}, g_{a b, c}, g_{a b, c d} ; \phi^{J}, \phi_{, a}^{J}, \phi_{, a b}^{J}\right),
\end{gathered}
$$

where $\delta \mathcal{L} / \delta A$ is the variation of $\mathcal{L}$ with respect to a field $A$.

Let us consider the variation of the action under an infinitesimal coordinate transformation, $x^{a} \rightarrow x^{a}+\xi^{a}$, which is given by

$$
\delta \int d^{4} x \mathcal{L}=2 \int d^{4} x \sqrt{-g}\left(\nabla_{b} \mathcal{G}^{a b}-\frac{1}{2} \mathcal{E}_{I} \nabla^{a} \phi^{I}\right) \xi_{a} .
$$

It follows from the assumption of $\mathcal{L}$ being a scalar density that eq. (2.4) vanishes identically, implying that the integrand itself vanishes since $\xi_{a}$ may be chosen arbitrarily. Thus, the identity

$$
\nabla_{b} \mathcal{G}^{a b}=\frac{1}{2} \mathcal{E}_{I} \nabla^{a} \phi^{I}
$$

holds. In the case of pure Einstein gravity this identity reduces to the well-known contracted Bianchi identity, $\nabla_{a} G^{a b}=0$. In this sense, the identity (2.5) may be regarded as a generalization of the Bianchi identity. We are trying to construct the most general biscalar-tensor theory with second-order field equations using the identity (2.5). Because both $\mathcal{G}^{a b}$ and $\mathcal{E}_{I}$ are assumed to be of second order in derivatives, the left-hand side of eq. (2.5) would yield third derivatives in general while the right-hand side contains at most second derivatives. This indicates that the left-hand side of eq. (2.5) must be free from third derivatives.

The construction of the most general second-order equations of motion for bi-scalartensor theories is divided into two parts: we first determine the most general secondorder rank-2 tensor whose divergence remains of second order. After that, we impose the identity (2.5) on the rank-2 tensor to constrain its form.

\subsection{Second-order rank-2 tensor whose divergence is of second order}

In this subsection we construct the most general second-order rank-2 tensor, $\widetilde{\mathcal{G}}^{a b}$, whose divergence is also of second order. The conditions that $\nabla_{a} \widetilde{\mathcal{G}}^{a b}$ has no third derivatives can be expressed as

$$
\begin{aligned}
& \frac{\partial \nabla_{b} \widetilde{\mathcal{G}}^{a b}}{\partial g_{c d, e f g}}=0, \\
& \frac{\partial \nabla_{b} \widetilde{\mathcal{G}}^{a b}}{\partial \phi_{, c d e}^{I}}=0 .
\end{aligned}
$$

Using the chain rule, $\nabla_{b} \widetilde{\mathcal{G}}^{a b}$ is rewritten as

$$
\begin{aligned}
\nabla_{b} \widetilde{\mathcal{G}}^{a b}= & \widetilde{\mathcal{G}}^{a b ; c d, e f} g_{c d, e f b}+\widetilde{\mathcal{G}}^{a b ; c d, e} g_{c d, e b}+\widetilde{\mathcal{G}}^{a b ; c d} g_{c d, b}+\widetilde{\mathcal{G}}^{a b ; c d} \phi_{, c d b}^{I}+\widetilde{\mathcal{G}}^{a b ; c}{ }_{I} \phi_{, c b}^{I} \\
& +\widetilde{\mathcal{G}}^{a b ;}{ }_{I} \phi_{, b}^{I}+\widetilde{\mathcal{G}}^{b c} \Gamma_{b c}^{a}+\widetilde{\mathcal{G}}^{a b} \Gamma_{b c}^{c},
\end{aligned}
$$


where $\Gamma_{b c}^{a}$ are the Christoffel symbols. With the help of eq. (2.8), we can show that the conditions (2.6) and (2.7) are equivalent to

$$
\begin{aligned}
\widetilde{\mathcal{G}}^{a b ; c d, e f}+\widetilde{\mathcal{G}}^{a e ; c d, f b}+\widetilde{\mathcal{G}}^{a f ; c d, b e} & =0, \\
\widetilde{\mathcal{G}}^{a b ; c d}+\widetilde{\mathcal{G}}^{a c ; d b}+\widetilde{\mathcal{G}}_{I}^{a d ; b c}=0 & =0
\end{aligned}
$$

respectively. From the "invariance identity" (see refs. [31, 32]), we have

$$
\widetilde{\mathcal{G}}^{a b ; c d, e f}+\widetilde{\mathcal{G}}^{a b ; c e, f d}+\widetilde{\mathcal{G}}^{a b ; c f, d e}=0 .
$$

By repeated use of eqs. (2.9), (2.10), and (2.11), we obtain

$$
\begin{aligned}
\widetilde{\mathcal{G}}^{a b ; c d, e f} & =\widetilde{\mathcal{G}}^{c d ; a b, e f}=\widetilde{\mathcal{G}}^{e f ; c d, a b}, \\
\widetilde{\mathcal{G}}^{a b ; c d} & =\widetilde{\mathcal{G}}_{I}^{c d ; a b} .
\end{aligned}
$$

For convenience, we now introduce the notion of property $S$ following ref. [11]. A quantity $A^{a_{1} a_{2} \ldots a_{2 n-1} a_{2 n}}$ is said to have property $S$ if it satisfies the following conditions: (i) it is symmetric in $\left(a_{2 \ell-1}, a_{2 \ell}\right)$ for $\ell=1,2, \ldots, n$; (ii) it is symmetric under the interchange of any two pairs $\left(a_{2 \ell-1}, a_{2 \ell}\right)$ and $\left(a_{2 m-1}, a_{2 m}\right)$ for $\ell, m=1,2, \ldots, n$; (iii) it vanishes if any three of four indices, $\left(a_{2 \ell-1}, a_{2 \ell}\right)$ and $\left(a_{2 m-1}, a_{2 m}\right)$ for $\ell, m=1,2, \ldots, n$, are symmetrized. It is shown in Corollary 2.1 of ref. [11] that $A^{a_{1} a_{2} \ldots a_{2 n-1} a_{2 n}}$ vanishes if $A^{a_{1} a_{2} \ldots a_{2 n-1} a_{2 n}}$ has property $S$ and $n>4$ in four-dimensional spacetime.

Let us introduce the quantity $B^{a_{1} a_{2} \ldots a_{4 n+2 m+1} a_{4 n+2 m+2}}$ defined by

$$
B^{a_{1} a_{2} \ldots a_{4 n+2 m+1} a_{4 n+2 m+2}} \equiv \Pi_{i=1}^{n}\left(\frac{\partial}{\partial g_{a_{4 i-1} a_{4 i}, a_{4 i+1} a_{4 i+2}}}\right) \Pi_{j=1}^{m}\left(\frac{\partial}{\partial \phi_{, a_{4 n+2 j+1} a_{4 n+2 j+2}}^{I_{j}}}\right) \widetilde{\mathcal{G}}^{a_{1} a_{2}} .
$$

This is a $n$-th derivative with respect to $g_{a_{i} a_{i+1}, a_{i+2} a_{i+3}}$ and $m$-th derivative with respect to $\phi_{, a_{i} a_{i+1}}^{I}$ of $\widetilde{\mathcal{G}}^{a b}$. Using eqs. (2.9)-(2.13), one can easily check that $B^{a_{1} a_{2} \ldots a_{4 n+2 m+1} a_{4 n+2 m+2}}$ has property $S$. Then, Corollary 2.1 of ref. [11] implies that $B^{a_{1} a_{2} \ldots a_{4 n+2 m+1} a_{4 n+2 m+2}}$ vanishes for $2 n+m \geq 4$, leading to the following three sets of identities:

$$
\begin{aligned}
& \frac{\partial}{\partial g_{c d, e f}} \frac{\partial}{\partial g_{i j, k l}} \widetilde{\mathcal{G}}^{a b}=0, \\
& \frac{\partial}{\partial g_{c d, e f}} \frac{\partial}{\partial \phi_{, i j}^{I}} \frac{\partial}{\partial \phi_{, k l}^{J}} \widetilde{\mathcal{G}}^{a b}=0, \\
& \frac{\partial}{\partial \phi_{, c d}^{I}} \frac{\partial}{\partial \phi_{, e f}^{J}} \frac{\partial}{\partial \phi_{, i j}^{K}} \frac{\partial}{\partial \phi_{, k l}^{L}} \widetilde{\mathcal{G}}^{a b}=0 .
\end{aligned}
$$

By integrating eqs. (2.15)-(2.17), we can determine the form of the gravitational field equations. First, integrating eq. (2.15) yields

$$
\widetilde{\mathcal{G}}^{a b}=\widetilde{\xi}^{a b c d e f} g_{c d, e f}+\widetilde{\xi}^{a b}=\xi^{a b c d e f} R_{c d e f}+\xi^{a b},
$$


where $\xi^{a b c d e f}$ and $\xi^{a b}$ are functions of $g_{a b}, g_{a b, c}, \phi^{I}, \phi_{, a}^{I}$, and $\phi_{, a b}^{I}$. Note that we have used the identity

$$
\tilde{\xi}^{a b c d e f} g_{c d, e f}=\frac{2}{3} \tilde{\xi}^{a b c d e f} R_{e c d f}+\bar{\xi}^{a b}
$$

at the second equality of eq. (2.18), where $\bar{\xi}^{a b}$ are functions of $g_{a b}, g_{a b, c}, \phi^{I}, \phi_{, a}^{I}$, and $\phi_{, a b}^{I}$. It can be seen that $\xi^{a b c d e f}$ and $\xi^{a b}$ have property $S$. Substituting eq. (2.18) into eq. (2.16) and integrating it, we obtain

$$
\widetilde{\mathcal{G}}^{a b}=\xi_{I}^{a b c d e f g h} R_{c d e f} \phi_{\mid g h}^{I}+\xi^{a b c d e f} R_{c d e f}+\xi^{a b},
$$

where $\xi^{a b c d e f g h}$ and $\xi_{I}^{a b c d e f g h}$ are functions of $g_{a b}, g_{a b, c}, \phi^{I}$, and $\phi_{, a}^{I}$, while $\xi^{a b}$ are functions of $g_{a b}, g_{a b, c}, \phi^{I}, \phi_{, a}^{I}$, and $\phi_{, a b}^{I}$. Here again it can be seen that $\xi^{a b c d e f g h}, \xi_{I}^{a b c d e f g h}$, and $\xi^{a b}$ have property $S$. Repeating the same procedure and integrating eq. (2.17) give

$$
\begin{aligned}
\widetilde{\mathcal{G}}^{a b}= & \xi_{I}^{a b c d e f g h} R_{c d e f} \phi_{\mid g h}^{I}+\xi_{I J K}^{a b c d e f g h} \phi_{\mid c d}^{I} \phi_{\mid e f}^{J} \phi_{\mid g h}^{K}+\xi^{a b c d e f} R_{c d e f} \\
& +\xi_{I J}^{a b c d e f} \phi_{\mid c d}^{I} \phi_{\mid e f}^{J}+\xi_{I}^{a b c d} \phi_{\mid c d}^{I}+\xi^{a b}
\end{aligned}
$$

where all of the above $\xi$ tensors are composed of $g_{a b}, g_{a b, c}, \phi^{I}$, and $\phi_{, a}^{I}$, and have property $S$. Although our final goal is to determine the most general equations of motion for the bi-scalar-tensor theory, the equations given up to this point hold irrespective of the number of the scalar fields.

Our remaining task in this subsection is to construct explicitly all the possible $\xi$ tensors that have property $S$ and are composed of $g_{a b}, g_{a b, c}, \phi^{I}$, and $\phi_{, a}^{I}$. For this purpose, we can use $\phi^{I \mid a}, g^{a b}$, and the totally antisymmetric tensor $\varepsilon^{a b c d}$ as building blocks, from which the $\xi$ tensors are built by taking their products and linear combinations appropriately. There is no elegant way, and what we will do is to exhaust all the possible combinations of those building blocks yielding the $\xi$ tensors. Let us begin with the simplest one, $\xi^{a b}$. It is not difficult to find that the following one is the most general symmetric rank-2 tensor composed of $\phi^{I}, \phi_{\mid a}^{I}, g^{a b}$, and $g^{a b, c}$ :

$$
\xi^{a b}=a\left(\phi^{I}, X^{J K}\right) g^{a b}+b_{I J}\left(\phi^{I}, X^{J K}\right) \phi^{I \mid a} \phi^{J \mid b}
$$

where $a\left(\phi^{I}, X^{J K}\right)$ and $b^{I J}\left(\phi^{I}, X^{J K}\right)$ are arbitrary functions of $\phi^{I}$ and $X^{J K}$, and $b^{I J}$ has the symmetric property $b^{I J}=b^{J I} .^{2}$ Here, we have used, for the first time in this derivation, the assumption that the number of the scalar fields is two, which greatly simplifies the expressions of $\xi$ tensors and the following procedure. Without this restriction we would have for example the term such as $c_{I J K L}\left(\varepsilon^{a c d e} \phi_{\mid c}^{I} \phi_{\mid d}^{J} \phi_{\mid e}^{K} \phi^{L \mid b}+\varepsilon^{b c d e} \phi_{\mid c}^{I} \phi_{\mid d}^{J} \phi_{\mid e}^{K} \phi^{L \mid a}\right)$ in $\xi^{a b}$, where $c_{I J K L}$ is arbitrary functions of $\phi^{I}$ and $X^{I J}$, and totally anti-symmetric in $I, J$ and

\footnotetext{
${ }^{2}$ One might consider other rank-2 tensors such as $\varepsilon^{a b c d} \phi_{\mid c}^{I} \phi_{\mid d}^{J}$, but this tensor is excluded because it is not symmetric in $a, b$.
} 
$K$. In a similar manner, we work out $\xi_{I}^{a b c d}$ :

$$
\begin{aligned}
\xi_{I}^{a b c d}= & a_{I}\left(g^{a c} g^{b d}+g^{a d} g^{b c}-2 g^{a b} g^{c d}\right) \\
& +b_{I J K}\left[g^{a c} \phi^{J \mid b} \phi^{K \mid d}+g^{a d} \phi^{J \mid b} \phi^{K \mid c}+g^{b d} \phi^{J \mid a} \phi^{K \mid c}\right. \\
& \left.+g^{b c} \phi^{J \mid a} \phi^{K \mid d}-2\left(g^{a b} \phi^{J \mid c} \phi^{K \mid d}+g^{c d} \phi^{J \mid a} \phi^{K \mid b}\right)\right] \\
& +c_{I J K L M}\left(\phi^{J \mid a} \phi^{K \mid c} \phi^{L \mid b} \phi^{M \mid d}+\phi^{J \mid a} \phi^{K \mid d} \phi^{L \mid b} \phi^{M \mid c}-2 \phi^{J \mid a} \phi^{K \mid b} \phi^{L \mid c} \phi^{M \mid d}\right) \\
& +d_{I J K L M}\left(\phi^{J \mid a} \phi^{K \mid c} \varepsilon^{b d e f} \phi_{\mid e}^{L} \phi_{\mid f}^{M}+\phi^{J \mid a} \phi^{K \mid d} \varepsilon^{b c e f} \phi_{\mid e}^{L} \phi_{\mid f}^{M}\right),
\end{aligned}
$$

where $a_{I}, b_{I J K}, c_{I J K L M}$, and $d_{I J K L M}$ are arbitrary functions of $\phi^{I}$ and $X^{J K}$ satisfying

$$
\begin{aligned}
b_{I J K} & =b_{I K J}, \\
c_{I J K L M} & =c_{I K J L M}=c_{I J K M L}=c_{I L M J K}, \\
d_{I J K L M} & =-d_{I K J L M}=-d_{I J K M L}=d_{I L M J K} .
\end{aligned}
$$

The explicit forms of $\xi^{a b c d e f}, \xi_{I J}^{a b c d e f}, \xi^{a b c d e f g h}$, and $\xi_{I}^{a b c d e f g h}$ for the bi-scalar case are given in appendix A. Substituting all the $\xi$ tensors into eq. (2.21) and rearranging the equation, we arrive at the most general second-order rank-2 tensor $\widetilde{\mathcal{G}}^{a b}$ whose divergence is also of second order,

$$
\begin{aligned}
\widetilde{\mathcal{G}}_{b}^{a}= & A \delta_{b}^{a}+B_{I J} \phi^{I \mid a} \phi_{\mid b}^{J}+C_{I} \delta_{b d}^{a c} \phi_{\mid c}^{I \mid d}+D_{I J K} \delta_{b d f}^{a c e} \phi_{\mid c}^{I} \phi^{J \mid d} \phi_{\mid e}^{K \mid f}+E_{I J K L M} \delta_{b d f h}^{a c e g} \phi_{\mid c}^{I} \phi^{J \mid d} \phi_{\mid e}^{K} \phi^{L \mid f} \phi_{\mid g}^{M \mid h} \\
& +F_{I J K L M} \delta_{b d f h}^{a c e g}\left(\varepsilon_{c e p q} \phi^{I \mid p} \phi^{J \mid q} \phi^{K \mid d} \phi^{L \mid f}+\phi_{\mid c}^{I} \phi_{\mid e}^{J} \varepsilon^{d f p q} \phi_{\mid p}^{K} \phi_{\mid q}^{L}\right) \phi_{\mid g}^{M \mid h}+G_{I J} \delta_{b d f}^{a c e} \phi_{\mid c}^{I \mid d} \phi_{\mid e}^{J \mid f} \\
& +H_{I J K L} \delta_{b d f h}^{a c e g} \phi_{\mid c}^{I} \phi^{J \mid d} \phi_{\mid e}^{K \mid f} \phi_{\mid g}^{L \mid h}+I \delta_{b d f}^{a c e} R_{c e}{ }^{d f}+J_{I J} \delta_{b d f h}^{a c e g} \phi_{\mid c}^{I} \phi^{J \mid d} R_{e g}{ }^{f h}+K_{I} \delta_{b d f h}^{a c e g} \phi_{\mid c}^{I \mid d} R_{e g}{ }^{f h} \\
& +L_{I J K} \delta_{b d f h}^{a c e g} \phi_{\mid c}^{I \mid d} \phi_{\mid e}^{J \mid f} \phi_{\mid g}^{K \mid h},
\end{aligned}
$$

where $A, B_{I J}, C_{I}, D_{I J K}, E_{I J K L M}, F_{I J K L M}, G_{I J}, H_{I J K L}, I, J_{I J}, K_{I}$, and $L_{I J K}$ are arbitrary functions of $\phi^{I}$ and $X^{I J}$, and they are subject to

$$
\begin{array}{rlrl}
B_{I J} & =B_{J I}, \quad D_{I J K}=D_{J I K}, & E_{I J K L M}=-E_{K J I L M}=-E_{I L K J M}=E_{J I L K M}, \\
G_{I J} & =G_{J I}, H_{I J K L}=H_{J I K L}=H_{I J L K}, & F_{I J K L M}=-F_{J I K L M}=-F_{I J L K M}=F_{J I L K M}, \\
J_{I J} & =J_{J I}, & L_{I J K} & =L_{J I K}=L_{I K J} .
\end{array}
$$

\subsection{Consequence of eq. (2.5)}

In the previous subsection we have obtained the most general second-order rank-2 tensor whose divergence remains of second order, $\widetilde{\mathcal{G}}^{a b}$. As can be seen, $\widetilde{\mathcal{G}}^{a b}$ involves many arbitrary functions. It turns out, however, that those functions are not completely independent in order for the equations of motion to be compatible with general covariance. In this subsection, we impose the identity (2.5) that arises due to general covariance, i.e., we require that the divergence of $\widetilde{\mathcal{G}}^{a b}$ is written as a product of $\phi^{I \mid a}$ and some scalar function. This procedure will reduce the number of the arbitrary functions. A straightforward calculation shows that 


$$
\begin{aligned}
\nabla^{b} \widetilde{\mathcal{G}}_{b}^{a}= & \mathcal{Q}_{I} \phi^{I \mid a}+\alpha_{I J} \delta_{b d f}^{a c e} \phi_{\mid c}^{I \mid d} \phi^{J \mid l} R_{e l}^{b f}+\beta_{I J} \delta_{b d f}^{a c e} \phi_{\mid l}^{I} \phi^{J \mid l b} R_{c e}^{d f}+\gamma_{I J K L} \delta_{b d f}^{a c e} \phi_{\mid l}^{K} \phi^{L \mid l b} \phi_{\mid c}^{I} \phi^{J \mid m} R_{e m}^{d f} \\
& +\epsilon_{I J K} \delta_{d f h m}^{a c e l} \phi_{\mid c}^{K \mid d} \phi_{\mid e}^{I \mid f} \phi^{J \mid g} R_{g l}{ }^{h m}+\mu_{I} \delta_{b d}^{a c} R_{c l}{ }^{b d} \phi^{I \mid l}+\nu_{I J K L} \delta_{b d f}^{a c e} \phi_{\mid l}^{K} \phi^{L \mid l b} \phi_{\mid c}^{I \mid d} \phi_{\mid e}^{J \mid f} \\
& +\omega_{I J K} \delta_{b d}^{a c} \phi_{\mid l}^{J} \phi^{K \mid l b} \phi_{\mid c}^{I \mid d}+\xi_{I J} \phi^{I \mid l} \phi_{\mid l}^{J \mid a}+\zeta_{I[J K]} \delta_{b d}^{a c} \phi_{\mid c}^{I} \phi^{J \mid l} \phi^{K \mid m} R_{l m}^{b d} \\
& +\iota_{I J K} \delta_{b d f h}^{a c e g} \phi_{\mid l}^{J} \phi^{K \mid l b} \phi_{\mid c}^{I \mid d} R_{e g}^{f h}+2 \eta_{I[J|K| L]} \delta_{b f h}^{a c e} \phi_{\mid c}^{I} \phi^{J \mid g} \phi_{\mid e}^{K \mid f} \phi^{L \mid l} R_{g l}^{b h} \\
& +\left(\lambda_{I J K L M}-\lambda_{I L M J K}\right) \delta_{b d}^{a c} \phi_{\mid e}^{L} \phi^{M \mid e b} \phi_{\mid c}^{I} \phi^{J \mid f} \phi_{\mid f}^{K \mid d}+\sigma_{I J K L M N} \delta_{b f h}^{a c g} \phi_{\mid l}^{M} \phi^{N \mid l b} \phi_{\mid c}^{I} \phi^{J \mid e} \phi_{\mid e}^{K \mid f} \phi_{\mid g}^{L \mid h} \\
& +\frac{3}{2} \tau_{I J K L M} \delta_{b d f h}^{a c e g} \phi^{L \mid l} \phi_{\mid l}^{M \mid b} \phi_{\mid c}^{I \mid d} \phi_{\mid e}^{J \mid f} \phi_{\mid g}^{K \mid h} \\
& +2 F_{I J K L M}\left(\varepsilon_{b d f h} \phi^{I \mid a} \phi^{J \mid g} \phi^{K \mid d} \phi^{L \mid f}+\varepsilon^{a c e g} \phi_{\mid c}^{I} \phi_{\mid e}^{J} \phi_{\mid b}^{K} \phi_{\mid h}^{L}\right) \phi^{M \mid l} R_{g l}{ }^{b h} \\
& +4 \varepsilon_{b d f h}\left(F_{I J K L M} \phi^{I \mid a} \phi^{J \mid g}\right)^{\mid b} \phi^{K \mid d} \phi^{L \mid f} \phi_{\mid g}^{M \mid h}+4 \varepsilon^{a c e g}\left(F_{I J K L M} \phi_{\mid c}^{I} \phi_{\mid e}^{J} \phi_{\mid b}^{K} \phi_{\mid h}^{L}\right)^{\mid b} \phi_{\mid g}^{M \mid h}, \quad(2.27)
\end{aligned}
$$

where the coefficients are functions of $\phi^{I}$ and $X^{J K}$ and defined as

$$
\begin{aligned}
\alpha_{I J}= & G_{I J}-2 J_{I J}+2 K_{I, J}-2 H_{K L I J} X^{K L}, \\
\beta_{I J}= & -I_{, I J}+J_{I J}-K_{J, I}+2 J_{K L, I J} X^{K L}, \\
\gamma_{I J K L}= & -2 J_{I J, K L}+H_{I K J L}, \\
\epsilon_{I J K}= & K_{(I, K) J}-\frac{3}{2} L_{K I J} \\
\mu_{I}= & \frac{1}{2} C_{I}+2 I_{, I}-\left(D_{J K I}+8 J_{J[K, I]}\right) X^{J K}+4 E_{J K L M I} X^{J K} X^{L M}, \\
\nu_{I J K L}= & -G_{I J, K L}+3 H_{K(I J L)}+2 H_{M N I J, K L} X^{M N}-3 L_{L I J, K}, \\
\omega_{I J K}= & -C_{I, J K}+2 D_{J(I K)}-2 G_{I K, J}+2\left(D_{L M I, J K}-4 H_{[J|L I K,| M]}\right) X^{L M} \\
& -16 E_{(I|J L M| K)} X^{L M}-8 E_{L M N O I, J K} X^{L M} X^{N O}, \\
\xi_{I J}= & -A_{, I J}+B_{I J}-C_{J, I}-4 D_{K[I|J,| L]} X^{K L} \\
& -8 E_{K L M N J, I} X^{K L} X^{M N}+16 E_{K I M N J, L} X^{K L} X^{M N}, \\
\zeta_{I J K}= & -\frac{1}{2} D_{I J K}-2 J_{I J, K}+4 E_{L M I J K} X^{L M}, \\
\eta_{I J K L}= & \frac{1}{2} H_{I J K L}, \\
\lambda_{I J K L M}= & \frac{1}{2} D_{I J K, L M}+H_{I J K M, L}-2 E_{M L I J K}-4 E_{N O I J K, L M} X^{N O}, \\
\sigma_{I J K L M N}= & H_{I J K L, M N}-H_{I M N L, J K}, \\
\tau_{I J K L M}= & -L_{[I|J K, L| M]}, \\
\iota_{I J K}= & -K_{[I, K] J} .
\end{aligned}
$$

We present the explicit form of $\mathcal{Q}_{I}$ in appendix B, though it is irrelevant to the following derivations.

In order for the right-hand side of eq. (2.27) to be proportional to $\phi^{I \mid a}$, all the terms that are not parallel to $\phi^{I \mid a}$ must vanish identically. We now derive the conditions for this following the procedure of ref. [11]. Let us first focus on the $\epsilon_{I J K}$ and $\iota_{I J K}$ terms in 
eq. (2.27), which are proportional to $\phi_{\mid b}^{I \mid a} \phi_{\mid d}^{J \mid c} R_{f h}{ }^{e g}$ :

$$
\nabla^{b} \widetilde{\mathcal{G}}_{b}^{a} \supset \epsilon_{I J K} \delta_{d f h k}^{a c e l} \phi^{K}{ }_{\mid c}^{\mid d} \phi_{\mid e}^{I \mid f} \phi^{J \mid g} R_{g l}{ }^{h k}+\iota_{I J K} \delta_{b d f h}^{a c e g} \phi^{J}{ }_{\mid l} \phi^{K \mid l b} \phi^{I \mid d}{ }_{\mid c} R_{e g}{ }^{f h}+\cdots .
$$

The coefficient of $\phi^{A}{ }_{, m n} \phi^{B}{ }_{, o p} g_{q r, s t}$ in this quantity can be extracted by taking a derivative with respect to $\phi^{A}{ }_{, m n} \phi^{B}{ }_{, o p} g_{q r, s t} \operatorname{as}^{3}$

$$
\begin{aligned}
\left(\nabla^{b} \widetilde{\mathcal{G}}_{b}^{a}\right)_{A}^{; m n ; o p} \underset{B}{m q r, s t}= & \left(\epsilon_{I J K} \delta_{d f h k}^{a c e l} \phi_{\mid c}^{K \mid d} \phi_{\mid e}^{I \mid f} \phi^{J \mid g} R_{g l}{ }^{h k}+\iota_{I J K} \delta_{b d f h}^{a c e g} \phi_{\mid l}^{J} \phi^{K \mid l b} \phi_{\mid c}^{I \mid d} R_{e g}{ }^{f h}\right)_{A}^{; m n ; o p ; q r, s t} \\
= & 2 \epsilon_{(A|J| B)} \delta_{d f h k}^{a c e l} \delta_{c}^{(m} g^{n) d} \delta_{e}^{(o} g^{p) f}\left(\phi^{J \mid(q} g^{r) k} \delta_{l}^{(s} g^{t) h}+g^{h(q} \delta_{l}^{r)} \phi^{J \mid(s} g^{t) k}\right) \\
& +2 \iota_{I J K} \delta_{b d f h}^{a c e g}\left(\delta_{A}^{K} \delta_{B}^{I} \phi^{J \mid(m} g^{n) b} \delta_{c}^{(o} g^{p) d}+\delta_{A}^{I} \delta_{B}^{K} \phi^{J \mid(o} g^{p) b} \delta_{c}^{(m} g^{n) d}\right) \delta_{e}^{(q} g^{r) h} \delta_{g}^{(s} g^{t) f} .
\end{aligned}
$$

Equation (2.30) must vanish when contracted with a vector $Y_{a}$ such that $Y_{a} \phi^{I \mid a}=0$ because eq. (2.5) implies that

$$
Y_{a}\left(\nabla^{b} \widetilde{\mathcal{G}}_{b}^{a}\right) \underset{A}{; m n} \underset{B}{m ; q p ; s t}=Y_{a}\left(\frac{1}{2} \mathcal{E}_{I} \phi^{I \mid a}\right)_{A}^{; m n ; o p ; q r, s t}=\frac{1}{2} Y_{a} \phi^{I \mid a} \mathcal{E}_{I}^{;} \underset{B}{; m n ; o p ; q r, s t}=0 .
$$

Thus, we obtain a constraint equation given by

$$
\begin{aligned}
& 2 \epsilon_{(A|J| B)} Y_{a} \delta_{d f h k}^{a c e l} \delta_{c}^{(m} g^{n) d} \delta_{e}^{(o} g^{p) f}\left(\phi^{J \mid(q} g^{r) k} \delta_{l}^{(s} g^{t) h}+g^{h(q} \delta_{l}^{r)} \phi^{J \mid(s} g^{t) k}\right) \\
+ & 2 \iota_{I J K} Y_{a} \delta_{b d f h}^{a c e g}\left(\delta_{A}^{K} \delta_{B}^{I} \phi^{J \mid(m} g^{n) b} \delta_{c}^{(o} g^{p) d}+\delta_{A}^{I} \delta_{B}^{K} \phi^{J \mid(o} g^{p) b} \delta_{c}^{(m} g^{n) d}\right) \delta_{e}^{(q} g^{r) h} \delta_{g}^{(s} g^{t) f}=0 .
\end{aligned}
$$

This constraint has eight free indices $m, n, o, p, q, r, s, t$, and its any component must be fulfilled. We first take the trace of eq. (2.32) by contracting with $g_{m n} g_{o p} g_{q r}$, giving

$$
-8 \epsilon_{(A|J| B)} \phi^{J(s} Y^{t)}+8 \iota_{(A|J| B)} \phi^{J(s} Y^{t)}=-8 \epsilon_{(A|J| B)} \phi^{J(s} Y^{t)}=0,
$$

where we have used $\iota_{(A|J| B)}=0$ which follows from the definition (2.28). This equation must be satisfied for any $\phi^{J \mid a}$, and therefore it is necessary to impose $\epsilon_{(A|J| B)}\left(=\epsilon_{A J B}\right)=0$. Further constraints can be derived from eq. (2.32) as follows. Let us project eq. (2.32) to the basis vectors $Y^{a}, \tilde{Y}^{a}, \phi^{I \mid a}(I=1,2)$ that satisfy

$$
Y_{a} Y^{a}=\tilde{Y}_{a} \tilde{Y}^{a}=1, \quad Y_{a} \tilde{Y}^{a}=0, \quad Y_{a} \phi^{I \mid a}=\tilde{Y}_{a} \phi^{I \mid a}=0 \quad \text { for } \quad I=1,2 .
$$

By contracting eq. (2.32) with $Z_{m} W_{n} V_{o} \phi_{\mid p}^{C} \phi_{\mid q}^{D} \phi_{\mid r}^{E} \phi_{\mid s}^{F} \phi_{\mid t}^{G}$, where $Z^{a}, W^{a}$, and $V^{a}$ are either $Y^{a}$ or $\tilde{Y}^{a}$, we find

$$
\begin{aligned}
0= & -4 \epsilon_{(A|J| B)} \delta_{b d}^{a c} Y_{a} V^{b}\left(Z_{c} W^{d}+W_{c} Z^{d}\right)\left(2 X^{(J \mid(D} X^{E)(F} X^{G) \mid C)}\right. \\
& \left.-X^{J(D} X^{E) C} X^{F G}-X^{D E} X^{C(F} X^{G) J}\right) \\
+ & 4 \iota_{A J B} \delta_{b d}^{a c} Y_{a} V^{b}\left(Z_{c} W^{d}+W_{c} Z^{d}\right) X^{J C}\left(X^{(D \mid(F} X^{G) \mid E)}-X^{D E} X^{F G}\right)
\end{aligned}
$$

${ }^{3}$ To obtain this result, we use the fact that derivatives of $\phi_{I, a b} \phi_{J, c d}$ and $R_{a b c d}$ are given by

$$
\begin{aligned}
\frac{\partial\left(\phi_{\mid a b}^{I} \phi_{\mid c d}^{J}\right)}{\partial \phi^{A}, m n \partial \phi^{B}, o p} & =\delta_{A}^{I} \delta_{(a}^{m} \delta_{b)}^{n} \delta_{B}^{J} \delta_{(c}^{o} \delta_{d)}^{p}+\delta_{A}^{J} \delta_{(c}^{m} \delta_{d)}^{n} \delta_{B}^{I} \delta_{(a}^{o} \delta_{b)}^{p}=\frac{1}{2}\left(\delta_{A}^{I} \delta_{B}^{J} D_{a c b d}^{m n o p}+\delta_{A}^{J} \delta_{B}^{I} D_{c a d b}^{m n o p}\right) \\
\frac{\partial R_{a b c d}}{\partial g_{q r, s t}} & =\frac{1}{4}\left(D_{a b c d}^{q r s t}+D_{c d a b}^{q r s t}-D_{a b d c}^{q r s t}-D_{b a c d}^{q r s t}\right)
\end{aligned}
$$

where $D_{a b c d}^{i j k l} \equiv 2 \delta_{(a}^{i} \delta_{d)}^{j} \delta_{(b}^{k} \delta_{c)}^{l}$. 
Using $\epsilon_{(A|J| B)}=0$ obtained in the previous step, we find $\iota_{A J B}=0$ as another constraint to be imposed.

Repeating a similar procedure for any other products of the second derivative terms in eq. (2.27), we find the following constraint equations for the coefficient functions:

$$
\begin{array}{r}
\alpha_{I J}=-2 \beta_{J I}, \quad \gamma_{I J K L}=-4 \eta_{I[J|L| K]}, \quad \alpha_{A I}\left[\delta_{I(C} X_{D) B}^{-1}-\delta_{I B} X_{C D}^{-1}\right]=4\left(\eta_{(C D) A B}-\eta_{(C|B A| D)}\right), \\
\epsilon_{I J K}=\iota_{I J K}=\omega_{I J K}=\lambda_{(I J) K L M}-\lambda_{(I|L M| J) K}=\mu_{I}=\zeta_{I[J K]}=\xi_{I J}=\tau_{I J K L M}=F_{I J K L M}=0, \\
\nu_{A C D B} X_{E F}^{-1}-\nu_{A B(E \mid C} X_{\mid F) D}^{-1}-2 \sigma_{(E F) C A D B}=0, \quad \nu_{B[A|K| C]}=\sigma_{E F[C A] D B}=0,
\end{array}
$$

where $X_{I J}^{-1}$ is the inverse matrix of $X^{I J}$.

The constraints (2.36) and (2.37) impose the following conditions on the functions appearing in eq. (2.25):

$$
\begin{aligned}
B_{I J}= & -2(\mathcal{F}+2 \mathcal{W})_{, I, J}+A_{, I J}+2 D_{(I|K| J), L} X^{K L}-16 E_{K(I|M N| J), L} X^{K L} X^{M N} \\
& -8\left(J_{K(I, J), L}-J_{K L, I, J}\right) X^{K L} \\
C_{I}= & -2(\mathcal{F}+2 \mathcal{W})_{, I}+2\left(D_{J K I}+8 J_{J[K, I]}\right) X^{J K}-8 E_{J K L M I} X^{J K} X^{L M} \\
F_{I J K L M}= & 0 \\
G_{I J}= & 2 J_{I J}-2 K_{(I, J)}+4 J_{K(I, J) L} X^{K L} \\
H_{I J K L}= & 2 J_{I J, K L} \\
K_{[I, J]}= & -2 J_{K[I, J] L} X^{K L} \\
K_{I, J K}= & K_{J, I K} \\
L_{I J K}= & \frac{2}{3} K_{(I, J K)} \\
I= & \frac{1}{2} \mathcal{F}+\mathcal{W}
\end{aligned}
$$

where $\mathcal{W}=\mathcal{W}\left(\phi^{I}\right)$, and $\mathcal{F}=\mathcal{F}\left(\phi^{I}, X^{J K}\right)$ is a function satisfying $\mathcal{F}_{, I J}=G_{I J}$, which is integrated to give

$$
\mathcal{F}=\int G_{I J} d X^{I J}=\int\left(2 J_{I J}-2 K_{I, J}+4 J_{K I, J L} X^{K L}\right) d X^{I J} .
$$

The conditions $\zeta_{I[J K]}=\omega_{I J K}=\lambda_{(I J) K L M}-\lambda_{(I|L M| J) K}=0$ in eq. (2.37) imply

$$
\begin{aligned}
D_{I[J K]}= & -4 J_{I[J, K]}+8 E_{L M I[J K]} X^{L M} \\
D_{I(J K)}= & \frac{1}{2} C_{J, I K}+G_{J K, I}+\left(-D_{L M J, I K}+4 H_{[I|L J K,| M]}\right) X^{L M} \\
& +8 E_{(J|I L M| K)} X^{L M}+4 E_{L M N O J, I K} X^{L M} X^{N O} \\
0= & \frac{1}{2} D_{I J K, L M}-\frac{1}{2} D_{(I|L M,| J) K}+H_{I J K M, L}-H_{(I|L M K,| J)} \\
& -2\left(E_{M L(I J) K}-E_{K(I J) L M}\right)-4\left(E_{N O(I J) K, L M}-E_{N O(I|L M,| J) K}\right) X^{N O},
\end{aligned}
$$


and eq. (2.38) implies

$$
\begin{aligned}
G_{I[J, K] L} & =0, \\
H_{I J K[L, M] N} & =0, \\
G_{(I J, K L)} & =3 H_{L(I J K)}+2 H_{L M(I J, K N)} X^{M N}-2 K_{(I, J K), L} .
\end{aligned}
$$

Equation (2.52) is nothing but the integrability condition which guarantees $\mathcal{F}_{, I J, K L}=$ $\mathcal{F}_{, K L, I J}$, and hence the integral (2.48) indeed exists.

\subsection{The most general second-order equation of motion}

Now we are at the final stage of deriving the most general second-order field equations of the bi-scalar-tensor theory. Substituting eqs. (2.39)-(2.47) into eq. (2.25), we at last obtain

$$
\begin{aligned}
\mathcal{G}_{b}^{a}= & A \delta_{b}^{a}+\left[-2 \mathcal{F}_{, I}-4 \mathcal{W}_{, I}+2\left(D_{J K I}+8 J_{J[K, I]}\right) X^{J K}-8 E_{J K L M I} X^{J K} X^{L M}\right] \delta_{b d}^{a c} \phi_{\mid c}^{I \mid d} \\
& +\left(-2 \mathcal{F}_{, I, J}-4 \mathcal{W}_{, I, J}+A_{, I J}+2 D_{I K J, L} X^{K L}\right. \\
& \left.-16 E_{K I M N J, L} X^{K L^{M}} X^{M N}-16 J_{K[I, L], J} X^{K L}\right) \phi^{(I \mid a} \phi_{\mid b}^{J J} \\
& +D_{I J K} \delta_{b d f}^{a c e} \phi_{\mid c}^{I} \phi^{J \mid d} \phi_{\mid e}^{K \mid f}+E_{I J K L M} \delta_{b d f h}^{a c e g} \phi_{\mid c}^{I} \phi^{J \mid d} \phi_{\mid e}^{K} \phi^{L \mid f} \phi_{\mid g}^{M \mid h} \\
& +\left(\frac{1}{2} \mathcal{F}+\mathcal{W}\right) \delta_{b d f}^{a c e} R_{c e}^{d f}+\mathcal{F}_{, I J} \delta_{b d f}^{a c e} \phi_{\mid c}^{I \mid d} \phi_{\mid e}^{J \mid f} \\
& +J_{I J} \delta_{b d f h}^{a c e g} \phi_{\mid c}^{I} \phi^{J \mid d} R_{e g}^{f h}+2 J_{I J, K L} \delta_{b d f h}^{a c e g} \phi_{\mid c}^{I} \phi^{J \mid d} \phi_{\mid e}^{K \mid f} \phi_{\mid g}^{L \mid h}+K_{I} \delta_{b d f h}^{a c e g} \phi_{\mid c}^{I \mid d} R_{e g}^{f h} \\
& +\frac{2}{3} K_{I, J K} \delta_{b d f h}^{a c e g} \phi_{\mid c}^{I \mid d} \phi_{\mid e}^{J \mid f} \phi_{\mid g}^{K \mid h} .
\end{aligned}
$$

This is the main result of this paper. The most general field equations for the singlescalar case [11] are reproduced as should be if one restricts the number of the scalar fields in eq. (2.55) to one. Note that one can eliminate $\mathcal{W}\left(\phi^{I}\right)$ from the above equation by redefining $\mathcal{F} \rightarrow \hat{\mathcal{F}}\left(\phi^{I}, X^{J K}\right)=\mathcal{F}+2 \mathcal{W}$. We can see that eqs. (2.50)-(2.54) do not reduce the number of the arbitrary functions because these are the relations between derivatives of the functions. In other words, these do not affect the structure of the field equations (2.55). As we will comment in the final section, these may, however, help us to check the integrability conditions for the field equations.

From the relation (2.5), the scalar-field equations of motion are found to be

$$
\mathcal{E}_{I}=2 \mathcal{Q}_{I}+\delta_{b d h m}^{c e g l}\left(-\gamma_{J I K L} \phi^{K \mid b} \phi_{\mid c}^{J} \phi_{\mid e}^{L \mid d} R_{g l}^{h m}+\frac{2}{3} \sigma_{J I K L M N} \phi_{\mid c}^{J} \phi^{M \mid b} \phi_{\mid e}^{K \mid d} \phi_{\mid g}^{L \mid h} \phi_{\mid l}^{N \mid m}\right) .
$$

\section{Comparison with the generalized multi-Galileon theory}

The covariant version of the multi-Galileon theory in the flat spacetime [23] was conjectured to be the most general multi-scalar-tensor theory with second-order field equations. However, later it was pointed out that this theory is not the most general one [25]. A counter-example is given by the multi-field DBI Galileons [27]. In this section, we compare the most general second-order field equations obtained in the previous section with the 
field equations of the generalized multi-Galileons, and identify the terms that are missing in the latter theory.

The action of the generalized multi-Galileons is given by [23]

$$
\begin{aligned}
\frac{1}{\sqrt{-g}} \mathcal{L}= & G_{2}-G_{3 I} \phi_{I \mid a}^{\mid a}+G_{4} R+G_{4, I J}\left(\phi_{\mid a}^{I \mid a} \phi_{\mid b}^{J \mid b}-\phi_{\mid a b}^{I} \phi^{J \mid a b}\right) \\
& +G_{5 I} G^{a b} \phi_{\mid a b}^{I}-\frac{1}{6} G_{5 I, J K}\left(\phi_{\mid a}^{I \mid a} \phi_{\mid b}^{J \mid b} \phi_{\mid c}^{K \mid c}-3 \phi_{\mid a}^{I \mid a} \phi_{\mid b}^{J \mid c} \phi_{\mid c}^{K \mid b}+2 \phi_{\mid a}^{I \mid b} \phi_{\mid b}^{J \mid c} \phi_{\mid c}^{K \mid a}\right)
\end{aligned}
$$

where $G_{2}, G_{3 I}, G_{4}$ and $G_{5 I}$ are arbitrary functions of $\phi^{I}$ and $X^{I J}$, and $G^{a b}$ is the Einstein tensor. The functions $G_{3 I, J K}, G_{4, I J, K L}, G_{5 I, J K}$ and $G_{5 I, J K, L M}$ are totally symmetric with respect to all of their indices, $I, J, K, L$ and $M$ in order for the field equations to be of second order. A straightforward calculation leads to the field equations for the generalized multi-Galileons,

$$
\begin{aligned}
E^{a b}(\mathcal{L})= & \left(-\frac{1}{2} G_{2}+G_{3(I, J)} X^{I J}-2 G_{4, I, J} X^{I J}\right) g^{a b}+\left(-\frac{1}{2} G_{2, I J}+G_{3(I, J)}-G_{4, I, J}\right) \phi^{I \mid a} \phi^{J \mid b} \\
& +\left(-X^{J K} G_{3 I J K}+G_{4, I}+2 X^{J K} G_{4 I J, K}\right) g^{l(a} \delta_{l d}^{b) c} \phi_{\mid c}^{I \mid d} \\
& +\left(-\frac{1}{2} G_{3 I J K}+2 G_{4 K(I, J)}-\frac{1}{2} G_{5 K, I, J}\right) g^{l(a} \delta_{l d f}^{b) c e} \phi_{\mid c}^{I} \phi^{J \mid d} \phi_{\mid e}^{K \mid f} \\
& -\frac{1}{4}\left(G_{4}-2 G_{4 I J} X^{I J}+G_{5(I, J)} X^{I J}\right) g^{l(a} \delta_{l d f}^{b) c e} R_{c e} d f \\
& +\left(\frac{1}{2} G_{4 I J}+X^{K L} G_{4 I J K L}-\frac{1}{2} G_{5(I, J)}-\frac{1}{2} X^{K L} G_{5 I J K, L}\right) g^{l(a} \delta_{l d f}^{b) c e} \phi_{\mid c}^{I \mid d} \phi_{\mid e}^{J \mid f} \\
& +\frac{1}{4}\left(G_{4 I J}-G_{5(I, J)}\right) g^{l(a} \delta_{l d f h}^{b) c e g} \phi_{\mid c}^{I} \phi^{J \mid d} R_{e g}^{f h} \\
& +\frac{1}{2}\left(G_{4 I J K L}-G_{5 K L(I, J)}\right) g^{l(a} \delta_{l d f h}^{b) c e g} \phi_{\mid c}^{I} \phi^{J \mid d} \phi_{\mid e}^{K \mid f} \phi_{\mid g}^{L \mid h} \\
& -\frac{1}{4} X^{J K} G_{5 I J K} g^{l(a} \delta_{l d f h}^{b) c e g} \phi_{\mid c}^{I \mid d} R_{e g}^{f h}-\frac{1}{6}\left(G_{5 I J K}+X^{L M} G_{5 I J K L M}\right) g^{l(a} \delta_{l d f h}^{b) c e g} \phi_{\mid c}^{I \mid d} \phi_{\mid e}^{J \mid f} \phi_{\mid g}^{K \mid h} .
\end{aligned}
$$

Comparing eq. (3.2) with eq. (2.55), it is easy to see the exact correspondence between each term. It is also found that terms corresponding to $E_{I J K L M}$ are lacking in the generalized multi-Galileon; this is a completely new term. We would, however, emphasize that, even setting $E_{I J K L M}=0$, eq. (2.55) covers a wider class of theories than the generalized multiGalileons. This fact is to be illustrated in a concrete example presented below. Note in passing that the coefficient functions of the above equation satisfy all the constraints (2.36)(2.38) found in the previous section.

The double-dual Riemann term deduced from the multi-field DBI Galileons,

$$
\mathcal{L}=\sqrt{-g} \delta_{I J} \delta_{K L} \delta_{b d f h}^{a c e g} \phi_{\mid a}^{I} \phi^{J \mid b} \phi_{\mid c}^{K} \phi^{L \mid d} R_{e g}^{f h},
$$

is not included in the Lagrangian of the generalized multi-Galileon theory [25]. One can however check that this term is actually contained in our theory. It is straightforward to derive the field equations from eq. (3.3):

$$
E^{a b}(\mathcal{L})=4 g^{l(a} \delta_{l d f h}^{b) c e g} X^{I[I} \phi_{\mid c}^{J]} \phi^{J \mid d} R_{e g}{ }^{f h}+8 g^{l(a} \delta_{l d f h}^{b) c e g} \delta_{I[J} \delta_{K] L} \phi_{\mid c}^{I} \phi^{J \mid d} \phi_{\mid e}^{K \mid f} \phi_{\mid g}^{L \mid h} .
$$

This is reproduced by setting $J_{I J}=2\left(\delta_{I J} \delta_{K L}-\delta_{I K} \delta_{J L}\right) X^{K L}$ in our field equations. 


\section{Candidate Lagrangian}

Having thus determined the most general second-order field equations of the bi-scalartensor theory, let us now explore the Lagrangian that gives the field equations we have derived. For the construction of the Lagrangian, we employ the same strategy as taken in ref. [11]. In the single scalar-field case, Horndeski found that the form of the Lagrangian can be guessed from the trace of the gravitational field equation. In the same way as in the single-field theory, we take the trace of the field equations of the bi-scalar-tensor theory (2.55) and arrive at the terms of the following form as a candidate Lagrangian:

$$
\begin{aligned}
& \mathcal{L}_{1}=\sqrt{-g} M^{(1)}{ }_{I} \phi_{\mid c}^{I \mid c}, \\
& \mathcal{L}_{2}=\sqrt{-g}\left(M^{(2)} \delta_{d f}^{c e} R_{c e}{ }^{d f}+2 M_{, I J}^{(2)} \delta_{d f}^{c e} \phi_{\mid c}^{I \mid d} \phi_{\mid e}^{J \mid f}\right), \\
& \mathcal{L}_{3}=\sqrt{-g} M_{I J K}^{(3)} \delta_{d f}^{c e} \phi_{\mid c}^{I} \phi^{J \mid d} \phi_{\mid e}^{K \mid f}, \\
& \mathcal{L}_{4}=\sqrt{-g}\left(M_{I}^{(4)} \delta_{d f h}^{c e g} \phi_{I}{ }_{\mid c}^{\mid d} R_{e g}{ }^{f h}+\frac{2}{3} M_{I, J K}^{(4)} \delta_{d f h}^{c e g} \phi_{\mid c}^{I \mid d} \phi_{\mid e}^{J \mid f} \phi_{\mid g}^{K \mid h}\right), \\
& \mathcal{L}_{5}=\sqrt{-g}\left(M_{I J}^{(5)} \delta_{d f h}^{c e g} \phi_{\mid c}^{I} \phi^{J \mid d} R_{e g}{ }^{f h}+2 M_{I J, K L}^{(5)} \delta_{d f h}^{c e g} \phi_{\mid c}^{I} \phi^{J \mid d} \phi_{\mid e}^{K \mid f} \phi_{\mid g}^{L \mid h}\right), \\
& \mathcal{L}_{6}=\sqrt{-g} M^{(6)}, \\
& \mathcal{L}_{7}=\sqrt{-g} M_{I J K L M}^{(7)} \delta_{d f h}^{c e g} \phi_{\mid c}^{I} \phi^{J \mid d} \phi_{\mid e}^{K} \phi^{L \mid f} \phi_{\mid g}^{M \mid h},
\end{aligned}
$$

where $M^{(1)}, M^{(2)}, M_{I J K}^{(3)}, M_{I}^{(4)}, M_{I J}^{(5)}, M^{(6)}$, and $M_{I J K L M}^{(7)}$ are arbitrary functions of $\phi^{I}$ and $X^{I J}$ satisfying

$$
\begin{aligned}
M_{I J K}^{(3)} & =M_{J I K}^{(3)}, \\
M_{I J}^{(5)} & =M_{J I}^{(5)}, \\
M_{I J K L M}^{(7)} & =-M_{K J I L M}^{(7)}=-M_{I L K J M}^{(7)}=M_{J I L K M}^{(7)} .
\end{aligned}
$$

In order to maintain the second-order equations of motion for the scalar fields, we have to impose extra conditions on these functions. For example, the Euler-Lagrange equation of $\mathcal{L}_{1}$ for the scalar field $\phi^{I}$ is given by

$$
\begin{aligned}
E_{I}\left(\mathcal{L}_{1}\right)= & M_{J, K I}^{(1)} \phi_{\mid c}^{J \mid c d} \phi_{\mid d}^{K}-M_{I, J K}^{(1)} \phi_{\mid c}^{J \mid d c} \phi_{\mid d}^{K}-2 M_{J, K I, L}^{(1)} X^{K L} \phi_{\mid c}^{J \mid c}+2 M_{I, J K, L}^{(1)} X_{\mid c}^{J K} \phi^{L \mid c} \\
& +M_{J, K I, L M}^{(1)} X^{L M \mid d} \phi_{\mid d}^{K} \phi_{\mid c}^{J \mid c}+M_{I, J K, L M}^{(1)} X_{\mid c}^{J K} X^{L M \mid c} \\
& +2 M_{(I, J)}^{(1)} \phi_{\mid c}^{J \mid c}-2 M_{I, J, K}^{(1)} X^{J K}+M_{J, K I}^{(1)} \phi_{\mid c}^{J \mid c} \phi_{\mid d}^{K \mid d}-M_{I, J K}^{(1)} \phi_{\mid c}^{J \mid d} \phi_{\mid d}^{K \mid c} .
\end{aligned}
$$

The two terms in the first line of eq. (4.11) are of third order, while the other terms are of second or first order. To eliminate the third-order derivatives, we therefore impose

$$
M_{[I, J] K}^{(1)}=0 .
$$

Performing the same analysis, we find that higher-derivative terms are removed by requiring that

$$
\begin{aligned}
M_{, I[J, K] L}^{(2)} & =0, \quad M_{I J[K, L] M}^{(3)}=0, \quad M_{[I, J] K}^{(4)}=0, \quad M_{I, J[K, L] M}^{(4)}=0, \\
M_{I J, K[L, M] N}^{(5)} & =0, \quad M_{I J K L[M, N] O}^{(7)}=0 .
\end{aligned}
$$


The Euler-Lagrange equations for the Lagrangian densities (4.1)-(4.7) are listed in appendix $\mathrm{C}$, from which one can see the relations between the functions appearing in eq. (2.55) and those in eqs. (4.1)-(4.7):

$$
\begin{aligned}
A= & M_{I, J}^{(1)} X^{I J}+4 M_{, I, J}^{(2)} X^{I J}-2 M_{I J K, L}^{(3)} X^{I J} X^{K L}-8\left(M_{I J, K, L}^{(5)}-M_{I L, J, K}^{(5)}\right) X^{I J} X^{K L} \\
& +\frac{1}{2} M^{(6)}+8 M_{I J K L M, N}^{(7)} X^{I J} X^{K L} X^{M N}, \\
D_{I J K}= & -\frac{1}{2} M_{(I, J) K}^{(1)}-4 M_{,(I, J) K}^{(2)}+\frac{3}{2} M_{(I J K)}^{(3)}+\left(M_{L(I J), M K}^{(3)}+M_{(I|L M,| J) K}^{(3)}-M_{I J L, K M}^{(3)}\right) X^{L M} \\
& -2 M_{K, I, J}^{(4)}+2\left(2 M_{K(I, J)}^{(5)}-M_{I J, K}^{(5)}\right)+4\left(2 M_{L(I, J), M K}^{(5)}-M_{I J, L, M K}^{(5)}\right) X^{L M} \\
& +12 M_{I J(K L M)}^{(7)} X^{L M}+8 M_{I J N L M, O K}^{(7)} X^{L M} X^{N O}, \\
E_{I J K L M}= & \frac{1}{8}\left(\delta_{I K}^{P Q} \delta_{J L}^{R S}+\delta_{J L}^{P Q} \delta_{I K}^{R S}\right)\left[-\frac{1}{2} M_{P R Q, S M}^{(3)}-4 M_{P R, Q, S M}^{(5)}+M_{P R Q S M}^{(7)}+M_{P R M S Q}^{(7)}+M_{P R Q M S}^{(7)}\right. \\
& \left.\quad-\left(M_{P R Q S N, O M}^{(7)}-2 M_{P R N S Q, O M}^{(7)}-2 M_{P N Q S O, R M}^{(7)}\right) X^{N O}\right], \\
\mathcal{F}+2 \mathcal{W}= & M^{(2)}-2 M_{, I J}^{(2)} X^{I J}-2 M_{I, J}^{(4)} X^{I J}+2 M_{I J}^{(5)} X^{I J}+4 M_{I K, J L}^{(5)} X^{I J} X^{K L}, \\
J_{I J}= & -\frac{1}{2} M_{, I J}^{(2)}-M_{(I, J)}^{(4)}+M_{I J}^{(5)}+\left(2 M_{K(I, J) L}^{(5)}-M_{I J, K L}^{(5)}\right) X^{K L}, \\
K_{I}= & -M_{J, K I}^{(4)} X^{J K} .
\end{aligned}
$$

In addition, comparing the $\phi^{(I \mid a} \phi^{J)}{ }_{\mid b}$ and $\delta_{b d}^{a c} \phi^{I \mid d}{ }_{c}^{\mid d}$ terms, we see that the following two conditions must be satisfied:

$$
\begin{aligned}
- & 2(\mathcal{F}+2 \mathcal{W})_{, I, J}+A_{I J}+2 D_{K(I J), L} X^{K L}+16 E_{K M N(I J), L} X^{K L} X^{M N}-8\left(J_{K(I, J), L}-J_{K L, I, J}\right) X^{K L} \\
= & M_{(I, J)}^{(1)}+2 M_{, I, J}^{(2)}-\left(M_{I J K, L}^{(3)}+2 M_{K L(I, J)}^{(3)}-2 M_{K(I J), L}^{(3)}\right) X^{K L} \\
& -4\left(M_{I J, K L}^{(5)}+M_{K L, I J}^{(5)}-2 M_{I K, J L}^{(5)}\right) X^{K L}+\frac{1}{2} M_{, I J}^{(6)} \\
& +8\left(M_{M N K L(I, J)}^{(7)}-2 M_{M N(K I), L}^{(7)}+M_{M N(I J) K, L}^{(7)}\right) X^{K L} X^{M N} \\
- & 2(\mathcal{F}+2 \mathcal{W})_{, K}+2\left(D_{I J K}+8 J_{I[J, K]}\right) X^{I J}-8 E_{I J L M K} X^{I J} X^{L M} \\
= & -M_{I, J K}^{(1)} X^{I J}-2\left(M_{, K}^{(2)}+2 M_{I, J K}^{(2)} X^{I J}\right)+3 M_{(I J K)}^{(3)} X^{I J}+2 M_{I J M, L K}^{(3)} X^{I L} X^{J M} .
\end{aligned}
$$

The Lagrangian is constructed by solving the above equations for $M^{(1)}, \ldots, M^{(7)}$, and unfortunately we have not accomplished this step yet. In appendix D, we review the construction of the Lagrangian for the single-scalar case. The lesson from the single-field Lagrangian is that we should probably integrate eq. (4.19) first to identify $M^{(4)}$ compatible with eq. (4.13). We have not yet succeeded even in solving these equations. In addition, it should be kept in mind that the terms we considered $\left(M^{(1)}, \ldots, M^{(7)}\right)$ might not be enough to construct the true Lagrangian. Those terms are simply inferred from the trace of the equations of motion, and in general there is no guarantee that they are enough though they happened to be so in the single scalar-field case. Albeit these difficulties, we believe that above calculations are useful to build the Lagrangian for our theory. We hope to report on this final part of the construction of the most general second-order bi-scalar-tensor theory in the near future. 


\section{Discussions and summary}

In this paper, we have reported our attempt to construct the bi-scalar generalization of the Horndeski theory in four-dimensional spacetime. Following Horndeski's method, we have succeeded in deriving all the possible terms appearing in the most general secondorder field equations for the bi-scalar tensor theory. We compared our field equations with those of the generalized multi-Galileon theory, and identified the terms that are not included in that theory. In particular, we confirmed that the double-dual Riemann term, which was shown to be missing in that theory [25], can be reproduced from our results by choosing the arbitrary functions appropriately. We have also discussed the construction of the Lagrangian yielding the field equations we found. For the construction, we have taken Horndeski's approach based on the trace of the field equations as a candidate Lagrangian. From the Euler-Lagrange equations we have obtained several differential equations for the functions in the Lagrangian, though we could not solve them to give explicit forms of the functions. In fact, it is still unclear whether or not the candidates of the Lagrangian we proposed suffice to generate all the terms in our most general field equations.

As discussed e.g. in ref. [15], we have to impose the integrability conditions on the field equations in order to ensure the existence of a corresponding Lagrangian. The integrability conditions are summarized as

$$
\begin{aligned}
\frac{\delta \sqrt{-g} \mathcal{G}^{\mu \nu}(x)}{\delta g_{\rho \lambda}(y)}-\frac{\delta \sqrt{-g} \mathcal{G}^{\rho \lambda}(y)}{\delta g_{\mu \nu}(x)} & =0, \\
\frac{\delta \sqrt{-g} \mathcal{G}^{\mu \nu}(x)}{\delta \phi^{I}(y)}-\frac{\delta \sqrt{-g} \mathcal{E}_{I}(y)}{\delta g_{\mu \nu}(x)} & =0, \\
\frac{\delta \sqrt{-g} \mathcal{E}_{I}(x)}{\delta \phi^{J}(y)}-\frac{\delta \sqrt{-g} \mathcal{E}_{J}(y)}{\delta \phi^{I}(x)} & =0,
\end{aligned}
$$

where $\delta / \delta A$ denotes variation with respect to a field $A$. Surprisingly, Horndeski found the corresponding Lagrangian in a rather heuristic way without using the integrability conditions explicitly. This implies that, in the single scalar case, those conditions are automatically satisfied and do not give rise to any extra constraints on the functions in the field equations. It is unclear that this property persists in theories with multiple scalar fields, and we have not been able to determine the corresponding Lagrangian completely. The integrability conditions might give us some clues to accomplish this procedure. We hope to report the result obtained from such an approach in the near future.

\section{Acknowledgments}

We would like to thank Xian Gao, Emir Gümrükçüoğlu, Hideo Kodama, Shinji Mukohyama, Vishagan Sivanesan, and Yi Wang for fruitful discussions and useful comments. S.O. was supported by JSPS Grant-in-Aid for Scientific Research No. 25-9997. N.T. was supported by the European Research Council grant no. ERC-2011-StG 279363-HiDGR. This work was supported in part by the JSPS Grant-in-Aid for Scientific Research Nos. 24740161 (T.K.), 25287054 and 26610062 (M.Y.). 


\section{A The $\xi$ tensors}

In this appendix, we show the expressions for the $\xi$ tensors introduced in section 2.2 to construct eq. (2.25). Expressions of $\xi^{a b}$ and $\xi_{I}^{a b c d}$ are given by eqs. (2.22) and (2.23), and we need to construct the other $\xi$ tensors appearing in eq. (2.21), i.e., $\xi^{a b c d e f}, \xi_{I J}^{a b c d e f}, \xi^{a b c d e f g h}$, and $\xi_{I}^{a b c d e f g h}$. As described in section 2.2, the $\xi$ tensors are constructed by taking the products and linear combinations of $\phi^{I \mid a}, g^{a b}$ and $\varepsilon^{a b c d}$ that enjoy property $S$, and we need to find all such $\xi$ tensors to construct the most general second-order tensor whose divergence remains of second order. In doing so, we find that not all of the $\xi$ tensors give nontrivial contributions to eq. (2.21) because there is some degeneracy and the identical terms appear from more than one $\xi$ tensor. Those $\xi$ tensors that give nontrivial contributions to eq. (2.21) are summarized as

$$
\begin{aligned}
\xi^{a b c d e f}= & \hat{a}_{I J}\left(\epsilon^{\text {Iace }} \epsilon^{J b d f}+\epsilon^{\text {Ibce }} \epsilon^{J a d f}+\epsilon^{\text {Iade }} \epsilon^{J b c f}+\epsilon^{\text {Ibde }} \epsilon^{J a c f}\right) \\
& +\hat{b} g_{g h}\left(\varepsilon^{a c e g} \varepsilon^{b d f h}+\varepsilon^{b c e g} \varepsilon^{a d f h}+\varepsilon^{a d e g} \varepsilon^{b c f h}+\varepsilon^{b d e g} \varepsilon^{a c f h}\right. \\
& \left.+\varepsilon^{a c f g} \varepsilon^{b d e h}+\varepsilon^{b c f g} \varepsilon^{a d e h}+\varepsilon^{a d f g} \varepsilon^{b c e h}+\varepsilon^{b d f g} \varepsilon^{a c e h}\right), \\
\xi_{I J}^{a b c d e f}= & \hat{a}_{I J K L}\left(\epsilon^{K a c e} \epsilon^{L b d f}+\epsilon^{K b c e} \epsilon^{\text {Ladf }}+\epsilon^{K a d e} \epsilon^{L b c f}+\epsilon^{K b d e} \epsilon^{L a c f}\right) \\
& +\hat{b}_{I J} g_{g h}\left(\varepsilon^{a c e g} \varepsilon^{b d f h}+\varepsilon^{b c e g} \varepsilon^{a d f h}+\varepsilon^{a d e g} \varepsilon^{b c f h}+\varepsilon^{b d e g} \varepsilon^{a c f h}\right. \\
& \left.+\varepsilon^{a c f g} \varepsilon^{b d e h}+\varepsilon^{b c f g} \varepsilon^{a d e h}+\varepsilon^{a d f g} \varepsilon^{b c e h}+\varepsilon^{b d f g} \varepsilon^{a c e h}\right), \\
\xi^{a b c d e f g h}= & \hat{a}\left(\varepsilon^{a c e g} \varepsilon^{b d f h}+\varepsilon^{b c e g} \varepsilon^{a d f h}+\varepsilon^{a d e g} \varepsilon^{b c f h}+\varepsilon^{b d e g} \varepsilon^{a c f h}\right. \\
& \left.+\varepsilon^{a c f g} \varepsilon^{b d e h}+\varepsilon^{b c f g} \varepsilon^{a d e h}+\varepsilon^{a d f g} \varepsilon^{b c e h}+\varepsilon^{b d f g} \varepsilon^{a c e h}\right),
\end{aligned}
$$

where $\hat{a}, \hat{a}_{I J}, \hat{a}_{I J K L}, \hat{b}$, and $\hat{b}_{I J}$ are arbitrary functions of $\phi^{M}$ and $X^{N O}$ satisfying $\hat{a}_{I J}=\hat{a}_{J I}$ and $\hat{a}_{I J K L}=\hat{a}_{I J L K}$. Here we have defined $\epsilon^{I a b c}$ as

$$
\epsilon^{I a b c}=\varepsilon^{a b c d} \phi_{\mid d}^{I} .
$$

\section{B Explicit form of $\mathcal{Q}_{I}$}

The explicit form of $\mathcal{Q}_{I}$ is given by

$$
\mathcal{Q}_{I} \equiv \mathcal{Q}_{I}^{(A)}+\mathcal{Q}_{I}^{(B)}+\mathcal{Q}_{I}^{(C)}+\mathcal{Q}_{I}^{(D)}+\mathcal{Q}_{I}^{(E)}+\mathcal{Q}_{I}^{(G)}+\mathcal{Q}_{I}^{(H)}+\mathcal{Q}_{I}^{(I)}+\mathcal{Q}_{I}^{(J)}+\mathcal{Q}_{I}^{(K)}+\mathcal{Q}_{I}^{(L)},
$$

with

$$
\begin{aligned}
\mathcal{Q}_{I}^{(A)}= & A_{, I} \\
\mathcal{Q}_{I}^{(B)}= & -2 B_{I J, K} X^{J K}-B_{I J, K L} \phi_{\mid c}^{K} \phi^{L \mid c b} \phi_{\mid b}^{J}+B_{I J} \phi_{\mid b}^{J \mid b} \\
\mathcal{Q}_{I}^{(C)}= & C_{J, I} \phi_{\mid c}^{J \mid c} \\
\mathcal{Q}_{I}^{(D)}= & D_{J K L, I} \delta_{d f}^{c e} \phi_{\mid c}^{J} \phi^{K \mid d} \phi_{\mid e}^{L \mid f}+2 D_{I J K, L} X^{J L} \phi_{\mid c}^{K \mid c}+D_{I J K, L} \phi^{J \mid c} \phi_{\mid c d}^{K} \phi^{L \mid d} \\
& +D_{I J K, L M} \delta_{b f}^{c e} \phi_{\mid d}^{L} \phi^{M \mid d b} \phi_{\mid c}^{J} \phi_{\mid e}^{K \mid f}-D_{I J K} \delta_{b f}^{c e} \phi_{\mid c}^{J \mid b} \phi_{\mid e}^{K \mid f}-\frac{1}{2} D_{I J K} \delta_{b f}^{c e} \phi_{\mid c}^{J} \phi^{K \mid l} R_{e l}{ }^{b f},
\end{aligned}
$$




$$
\begin{aligned}
\mathcal{Q}_{I}^{(E)}= & E_{J K L M N, I} \delta_{d f h}^{c e g} \phi_{\mid c}^{J} \phi^{K \mid d} \phi_{\mid e}^{L} \phi^{M \mid f} \phi_{\mid g}^{N \mid h}-2 E_{L J K I M, N} \delta_{d f h}^{c e g} \phi_{\mid c}^{L} \phi^{J \mid d} \phi_{\mid e}^{K} \phi^{N \mid f} \phi_{\mid g}^{M \mid h} \\
& -2 E_{L J K I M, N O} \delta_{b f h}^{c e g} \phi_{\mid l}^{N} \phi^{O \mid l b} \phi_{\mid c}^{L} \phi_{\mid e}^{K} \phi^{J \mid f} \phi_{\mid g}^{M \mid h}+4 E_{L J K I M} \delta_{b f h}^{c e g} \phi_{\mid c}^{L \mid b} \phi_{\mid e}^{K} \phi^{J \mid f} \phi_{\mid g}^{M \mid h} \\
& +E_{L J K I M} \delta_{b f h}^{c e g} \phi_{\mid c}^{L} \phi_{\mid e}^{K} \phi^{J \mid f} \phi^{M \mid l} R_{g l}^{b h} \\
\mathcal{Q}_{I}^{(G)}= & G_{J K, I} \delta_{d f}^{c e} \phi_{\mid c}^{J \mid d} \phi_{\mid e}^{K \mid f} \\
\mathcal{Q}_{I}^{(H)}= & H_{J K L M, I} \delta_{d f h}^{c e g} \phi_{\mid c}^{J} \phi^{K \mid d} \phi_{\mid e}^{L \mid f} \phi_{\mid g}^{M \mid h}-H_{I J K L} \delta_{b f h}^{c e g} \phi_{\mid c}^{J \mid b} \phi_{\mid e}^{K \mid f} \phi_{\mid g}^{L \mid h} \\
& +2 H_{I J K L, M} X^{J M} \delta_{f h}^{e g} \phi_{\mid e}^{K \mid f} \phi_{\mid g}^{L \mid h}+2 H_{I J K L, M} \delta_{f h}^{c g} \phi_{\mid c}^{J} \phi^{M \mid e} \phi_{\mid e}^{K \mid f} \phi_{\mid g}^{L \mid h} \\
& -H_{I J K L} \delta_{b f h}^{c e g} \phi_{\mid c}^{J} \phi_{\mid e}^{K \mid f} \phi^{L \mid l} R_{g l}{ }^{b h}+H_{I J K L, M N} \delta_{b f h}^{c e g} \phi_{\mid l}^{M} \phi^{N \mid l b} \phi_{\mid c}^{J} \phi_{\mid e}^{K \mid f} \phi_{\mid g}^{L \mid h} \\
\mathcal{Q}_{I}^{(I)}= & I_{, I} \delta_{d f}^{c e} R_{c e}^{d f}, \\
\mathcal{Q}_{I}^{(J)}= & J_{J K, I} \delta_{d f h}^{c e g} \phi_{\mid c}^{J} \phi^{K \mid d} R_{e g}^{f h}+2 J_{I J, K} X^{J K} \delta_{f h}^{e g} R_{e g}^{f h}+2 J_{I J, K} \delta_{f h}^{c g} \phi_{\mid c}^{J} \phi^{K \mid e} R_{e g}{ }^{f h} \\
& +J_{I J, K L} \delta_{b f h}^{c e g} \phi_{\mid d}^{K} \phi^{L \mid d b} \phi_{\mid c}^{J} R_{e g}^{f h}-J_{I J} \delta_{b f h}^{c e g} \phi_{\mid c}^{J \mid b} R_{e g}{ }^{f h}, \\
\mathcal{Q}_{I}^{(K)}= & K_{J, I} \delta_{d f h}^{c e g} \phi_{\mid c}^{J \mid d} R_{e g}^{f h}-\frac{1}{2} K_{J, I K} \delta_{d f h m}^{c e g l} \phi_{\mid c}^{K \mid d} \phi_{\mid e}^{J \mid f} R_{g l}{ }^{h m}-\frac{1}{8} K_{I} \delta_{d f h m}^{c e g l} R_{c e}{ }^{d f} R_{g l}{ }^{h m} \\
\mathcal{Q}_{I}^{(L)}= & L_{J K L, I} \delta_{d f h}^{c e g} \phi_{\mid c}^{J \mid d} \phi_{\mid e}^{K \mid f} \phi_{\mid g}^{L \mid h}-\frac{1}{4} L_{L J K, I M} \delta_{d f h m}^{c e g l} \phi_{\mid c}^{M \mid d} \phi_{\mid e}^{L \mid f} \phi_{\mid g}^{J \mid h} \phi_{\mid l}^{K \mid m} .
\end{aligned}
$$

\section{Euler-Lagrange equations}

The explicit forms of the Euler-Lagrange equations from $\mathcal{L}_{1}-\mathcal{L}_{7}$ are given as follows:

$$
\begin{aligned}
& E^{a b}\left(\mathcal{L}_{1}\right)=M_{I, J}^{(1)}\left(\phi^{I \mid(a} \phi^{J \mid b)}+g^{a b} X^{I J}\right)-\frac{1}{2} M_{I, J K}^{(1)}\left(g^{l(b} \delta_{l d f}^{a) c e} \phi_{\mid c}^{I} \phi^{J \mid d} \phi_{\mid e}^{K \mid f}+2 X^{I J} g^{l(b} \delta_{l d}^{a) c} \phi_{\mid c}^{K \mid d}\right), \\
& E^{a b}\left(\mathcal{L}_{2}\right)=2 M_{, I, J}^{(2)}\left(\phi^{I \mid(a} \phi^{J \mid b)}+2 g^{a b} X^{I J}\right)-2\left(M_{, I}^{(2)}+2 M_{, K, I J}^{(2)} X^{J K}\right) g^{l(a} \delta_{l d}^{b) c} \phi_{\mid c}^{I \mid d} \\
& -\frac{1}{2} M_{, I J}^{(2)} g^{l(a} \delta_{l d f h}^{b) c e g} \phi_{\mid c}^{I} \phi^{J \mid d} R_{e g}^{f h}+\left(\frac{1}{2} M^{(2)}-M_{, I J}^{(2)} X^{I J}\right) g^{l(a} \delta_{l d f}^{b) c e} R_{c e}{ }^{d f} \\
& \text { - }\left(M_{, I J}^{(2)}+2 M_{, I J, K L}^{(2)} X^{K L}\right) g^{l(a} \delta_{l d f}^{b) c e} \phi_{\mid c}^{I \mid d} \phi_{\mid e}^{J \mid f}-M_{, I J, K L}^{(2)} g^{l(a} \delta_{l d f h}^{b) c e g} \phi_{\mid c}^{I} \phi^{J \mid d} \phi_{\mid e}^{K \mid f} \phi_{\mid g}^{L \mid h} \\
& -4 M_{, I, J K}^{(2)} g^{l(a} \delta_{l d f}^{b) c e} \phi_{\mid c}^{I} \phi^{J \mid d} \phi_{\mid e}^{K \mid f}, \\
& E^{a b}\left(\mathcal{L}_{3}\right)=-2 M_{I J K, L}^{(3)} X^{I J} X^{K L} g^{a b}-M_{I J K, L}^{(3)}\left(X^{K L} \phi^{I \mid(a} \phi^{J \mid b)}+2 X^{I J} \phi^{K \mid(a} \phi^{L \mid b)}+2 X^{L(I} \phi^{J) \mid(a} \phi^{K \mid b)}\right) \\
& +\frac{3}{2} M_{(I J K)}^{(3)}\left(g^{l(a} \delta_{l d f}^{b) c e} \phi_{\mid c}^{I} \phi^{J \mid d} \phi_{\mid e}^{K \mid f}+2 X^{I J} g^{l(a} \delta_{l d}^{b) c} \phi_{\mid c}^{K \mid d}\right) \\
& -\frac{1}{2} M_{I J K, L M}^{(3)} g^{l(a} \delta_{l d f h}^{b) c e g} \phi_{\mid c}^{I} \phi^{J \mid d} \phi_{\mid e}^{K} \phi^{L \mid f} \phi_{\mid g}^{M \mid h} \\
& +\left(M_{I L M, J K}^{(3)}-2 M_{I[J M], K L}^{(3)}\right) X^{L M} g^{l(a} \delta_{l f h}^{b) c g} \phi_{\mid c}^{I} \phi^{J \mid f} \phi_{\mid g}^{K \mid h} \\
& +2 M_{I J K, L M}^{(3)} X^{I L} X^{J K} g^{l(a} \delta_{l d}^{b) c} \phi_{\mid c}^{M \mid d}, \\
& E^{a b}\left(\mathcal{L}_{4}\right)=-M_{I, J K}^{(4)} X^{I J} g^{n(a} \delta_{n f h m}^{b) e g l} \phi_{\mid e}^{K \mid f} R_{g l}{ }^{h m}-M_{I, J}^{(4)} X^{I J} g^{l(a} \delta_{l f h}^{b) e g} R_{e g}{ }^{f h} \\
& -M_{I, J}^{(4)} g^{l(a} \delta_{l d f h}^{b) c e g} \phi_{\mid c}^{I} \phi^{J \mid d} R_{e g}{ }^{f h} \\
& -\frac{2}{3}\left(M_{I, J K, L M}^{(4)} X^{I J}+M_{K, L M}^{(4)}\right) g^{n(a} \delta_{n f h m}^{b) e g l} \phi_{\mid e}^{K \mid f} \phi_{\mid g}^{L \mid h} \phi_{\mid l}^{M \mid m}-2 M_{I, J, K}^{(4)} g^{l(a} \delta_{l d f}^{b) c e} \phi_{\mid c}^{J} \phi^{K \mid d} \phi_{\mid e}^{I \mid f} \\
& -2\left(M_{I, J}^{(4)}+M_{K, I J, L}^{(4)} X^{K L}\right) g^{l(a} \delta_{l d f}^{b) c e} \phi_{\mid c}^{I \mid d} \phi_{\mid e}^{J \mid f}-2 M_{I, K(J, L)}^{(4)} g^{l(a} \delta_{l d f h}^{b) c e g} \phi_{\mid c}^{J} \phi^{L \mid d} \phi_{\mid e}^{I \mid f} \phi_{\mid g}^{K \mid h} \text {, }
\end{aligned}
$$




$$
\begin{aligned}
E^{a b}\left(\mathcal{L}_{5}\right)= & \left(M_{I J}^{(5)}+2 M_{I K, J L}^{(5)} X^{K L}\right) X^{I J} g^{l(a} \delta_{l f h}^{b) e g} R_{e g}{ }^{f h} \\
& +\left[M_{I J}^{(5)}+\left(2 M_{I K, J L}^{(5)}-M_{I J, K L}^{(5)}\right) X^{K L}\right] g^{l(a} \delta_{l d f h}^{b) c e g} \phi_{\mid c}^{I} \phi^{J \mid d} R_{e g}{ }^{f h} \\
& +2\left[M_{I J}^{(5)}+\left(M_{K L, I J}^{(5)}+4 M_{I K, J L}^{(5)}\right) X^{K L}+2 M_{M N, K L, I J}^{(5)} X^{K M} X^{L N}\right] g^{l(a} \delta_{l d f}^{b) c e} \phi_{\mid c}^{I \mid d} \phi_{\mid e}^{J \mid f} \\
& +2\left[2 M_{I K, J L}^{(5)}+\left(2 M_{I M, J N, K L}^{(5)}-M_{I J, K L, M N}^{(5)}\right) X^{M N}\right] g^{l(a} \delta_{l d f h}^{b) c e g} \phi_{\mid c}^{I} \phi^{J \mid d} \phi_{\mid e}^{K \mid f} \phi_{\mid g}^{L \mid h} \\
& +2\left[2 M_{K I, J}^{(5)}-M_{I J, K}^{(5)}+2\left(2 M_{I L, J, M K}^{(5)}-M_{I J, L, M K}^{(5)}\right) X^{L M}\right] g^{l(a} \delta_{l d f}^{b) c e} \phi_{\mid c}^{I} \phi^{J \mid d} \phi_{\mid e}^{K \mid f} \\
& -16 M_{I[J, L], K}^{(5)} X^{I J} X^{K L} g^{a b}-4\left(M_{I J, K, L}^{(5)}+M_{K L, I, J}^{(5)}-2 M_{I K, J, L}^{(5)}\right) X^{K L} \phi^{I \mid(a} \phi^{J \mid b)} \\
& -4 M_{I J, K, L M}^{(5)} g^{l(a} \delta_{l d f h}^{b) c e g} \phi_{\mid c}^{I} \phi^{J \mid d} \phi_{\mid e}^{K} \phi^{L \mid f} \phi_{\mid g}^{M \mid h}, \\
E^{a b}\left(\mathcal{L}_{6}\right)= & \frac{1}{2} M^{(6)} g^{a b}+\frac{1}{2} M_{, I J}^{(6)} \phi^{I \mid(a} \phi^{J \mid b)}, \\
E^{a b}\left(\mathcal{L}_{7}\right)= & \left(M_{I J K L M}^{(7)}+M_{I J M L K}^{(7)}+M_{I J K M L}^{(7)}\right) g^{l(a} \delta_{l d f h}^{b) c e g} \phi_{\mid c}^{I} \phi^{J \mid d} \phi_{\mid e}^{K} \phi^{L \mid f} \phi_{\mid g}^{M \mid h} \\
& +4\left(M_{I J K L M}^{(7)}+M_{K L M I J}^{(7)}+M_{K L I M J}^{(7)}\right) X^{I J} g^{l(a} \delta_{l d f}^{b) c e} \phi_{\mid c}^{K} \phi^{L \mid d} \phi_{\mid e}^{M \mid f} \\
& +\left(-M_{I J K L N, O M}^{(7)}+2 M_{I J N L K, O M}^{(7)}+2 M_{I N K L O, J M}^{(7)}\right) X^{N O} g^{l(a} \delta_{l d f h}^{b) c e g} \phi_{\mid c}^{I} \phi^{J \mid d} \phi_{\mid e}^{K} \phi^{L \mid f} \phi_{\mid g}^{M \mid h} \\
& +8 M_{N L I J M, K O}^{(7)} X^{L M} X^{N O} g^{l(a} \delta_{l d f}^{b) c e} \phi_{\mid c}^{I} \phi^{J \mid d} \phi_{\mid e}^{K \mid f}+8 M_{I J K L M, N}^{(7)} X^{I J} X^{K L} X^{M N} g^{a b} \\
& +8\left(M_{I J K L M, N}^{(7)}-2 M_{I J(N K) M, L}^{(7)}+M_{I J M N K, L}^{(7)}\right) X^{I J} X^{K L} \phi^{M \mid(a} \phi^{N \mid b)} .
\end{aligned}
$$

\section{Construction of Lagrangian: single scalar-field case}

In this appendix we briefly review the construction of the Lagrangian for the most general single scalar-tensor theory with second-order equations of motion (see ref. [11] for more detail). The most general second order field equations in the single scalar-field case are given by

$$
\begin{aligned}
\mathcal{G}_{b}^{a}= & A \delta_{b}^{a}+\left(-2 \mathcal{F}^{\prime \prime}+\dot{A}+2 D^{\prime} X\right) \phi^{\mid a} \phi_{\mid b}+\left(-2 \mathcal{F}^{\prime}+2 D X\right) \delta_{b d}^{a c} \phi_{\mid c}^{\mid d} \\
& +D \delta_{b d f}^{a c e} \phi_{\mid c} \phi^{\mid d} \phi_{\mid e}^{\mid f}+\frac{1}{2} \mathcal{F} \delta_{b d f}^{a c e} R_{c e}{ }^{d f}+\dot{\mathcal{F}} \delta_{b d f}^{a c e} \phi_{\mid c}^{\mid d} \phi_{\mid e}^{\mid f} \\
& +J \delta_{b d f h}^{a c e g} \phi_{\mid c} \phi^{\mid d} R_{e g}^{f h}+2 \dot{J} \delta_{b d f h}^{a c e g} \phi_{\mid c} \phi^{\mid d} \phi_{\mid e}^{\mid f} \phi_{\mid g}^{\mid h}+K \delta_{b d f h}^{a c e g} \phi_{\mid c}^{\mid d} R_{e g}^{f h}+\frac{2}{3} \dot{K} \delta_{b d f h}^{a c e g} \phi_{\mid c}^{\mid d} \phi_{\mid e}^{\mid f} \phi_{\mid g}^{\mid h},
\end{aligned}
$$

where $\phi$ is a scalar field and $X=-(\partial \phi)^{2} / 2$. A prime ", " and a dot " " denote derivatives with respect to $\phi$ and $X$, respectively, and $A, D, J$, and $K$ are arbitrary functions of $\phi$ and $X$, while $\mathcal{F}$ is related to the other functions as

$$
\mathcal{F}=\int\left(2 J-2 K^{\prime}+4 \dot{J} X\right) d X+\mathcal{W}
$$


where $\mathcal{W}$ is an arbitrary function of $\phi$. As explained in the main text, candidates for the Lagrangian can be guessed from the trace of the field equations as

$$
\begin{aligned}
& \mathcal{L}_{1}=\sqrt{-g} M^{(1)} \phi_{\mid c}^{\mid c}, \\
& \mathcal{L}_{2}=\sqrt{-g}\left(M^{(2)} \delta_{d f}^{c e} R_{c e}{ }^{d f}+2 \dot{M}^{(2)} \delta_{d f}^{c e} \phi_{\mid c}^{\mid d} \phi_{\mid e}^{\mid f}\right), \\
& \mathcal{L}_{3}=\sqrt{-g} M^{(3)} \delta_{d f}^{c e} \phi_{\mid c} \phi^{\mid d} \phi_{\mid e}^{\mid f}, \\
& \mathcal{L}_{4}=\sqrt{-g}\left(M^{(4)} \delta_{d f h}^{c e g} \phi_{\mid c}^{\mid d} R_{e g}{ }^{f h}+\frac{2}{3} \dot{M}^{(4)} \delta_{d f h}^{c e g} \phi_{\mid c}^{\mid d} \phi_{\mid e}^{\mid f} \phi_{\mid g}^{\mid h}\right), \\
& \mathcal{L}_{5}=\sqrt{-g}\left(M^{(5)} \delta_{d f h}^{c e g} \phi_{\mid c} \phi^{\mid d} R_{e g}{ }^{f h}+2 \dot{M}^{(5)} \delta_{d f h}^{c e g} \phi_{\mid c} \phi^{\mid d} \phi_{\mid e}^{\mid f} \phi_{\mid g}^{\mid h}\right), \\
& \mathcal{L}_{6}=\sqrt{-g} M^{(6)} .
\end{aligned}
$$

By comparing the most general field equations (D.1) with the Euler-Lagrange equations obtained from (D.3)-(D.8), it can be seen that the free functions are related as

$$
\begin{aligned}
A & =M^{(1) \prime} X+4 M^{(2) \prime \prime} X-2 M^{(3) \prime} X^{2}+\frac{1}{2} M^{(6)}, \\
D & =-\frac{1}{2} \dot{M}^{(1)}-4 \dot{M}^{(2) \prime}+\frac{3}{2} M^{(3)}+\dot{M}^{(3)} X-2 M^{(4) \prime \prime}+2 M^{(5) \prime}+4 \dot{M}^{(5) \prime} X, \\
\mathcal{F} & =M^{(2)}-2 \dot{M}^{(2)} X-2 M^{(4) \prime} X+2 M^{(5)} X+4 \dot{M}^{(5)} X^{2}, \\
J & =-\frac{1}{2} \dot{M}^{(2)}-M^{(4) \prime}+M^{(5)}+\dot{M}^{(5)} X, \\
K & =-\dot{M}^{(4)} X, \\
-2 \mathcal{F}^{\prime \prime}+\dot{A}+2 D^{\prime} X & =M^{(1) \prime}+2 M^{(2) \prime \prime}-M^{(3) \prime} X+\frac{1}{2} \dot{M}^{(6)}, \\
-2 \mathcal{F}^{\prime}+2 D X & =-\dot{M}^{(1)} X-2\left(M^{(2) \prime}+2 \dot{M}^{(2)} X\right)+3 M^{(3)} X+2 \dot{M}^{(3)} X^{2} .
\end{aligned}
$$

In order to identify the Lagrangian, we need to solve the above equations for $M^{(1)}$ $M^{(6)}$. First, by integrating eq. (D.13) we obtain

$$
M^{(4)}=-\int \frac{K}{X} d X
$$

Substituting eq. (D.16) into eqs. (D.11) and (D.12), we then find

$$
\begin{aligned}
& M^{(2)}=-2 \int\left(M^{(4) \prime}-M^{(5)}-2 X \dot{M}^{(5)}\right) d X, \\
& M^{(5)}=-\int \frac{J}{X} d X .
\end{aligned}
$$

Finally, we can integrate eqs. (D.9), (D.10), (D.14), and (D.15) to identify $M^{(1)}, M^{(3)}$, and $M^{(6)}$ with the help of eqs. (D.16), (D.17), and (D.18), leading to

$$
\begin{aligned}
& M^{(1)}=-3\left(2 M^{(2) \prime}-X M^{(3)}\right), \\
& M^{(3)}=-2 \int \frac{D}{X} d X,
\end{aligned}
$$


and

$$
M^{(6)}=2 A+4 X M^{(2) \prime \prime}-2 M^{(3) \prime} .
$$

Open Access. This article is distributed under the terms of the Creative Commons Attribution License (CC-BY 4.0), which permits any use, distribution and reproduction in any medium, provided the original author(s) and source are credited.

\section{References}

[1] A.A. Starobinsky, A New Type of Isotropic Cosmological Models Without Singularity, Phys. Lett. B 91 (1980) 99 [INSPIRE].

[2] K. Sato, First Order Phase Transition of a Vacuum and Expansion of the Universe, Mon. Not. Roy. Astron. Soc. 195 (1981) 467 [inSPIRE].

[3] A.H. Guth, The Inflationary Universe: A Possible Solution to the Horizon and Flatness Problems, Phys. Rev. D 23 (1981) 347 [inSPIRE].

[4] WMAP collaboration, C.L. Bennett et al., Nine-Year Wilkinson Microwave Anisotropy Probe (WMAP) Observations: Final Maps and Results, Astrophys. J. Suppl. 208 (2013) 20 [arXiv: 1212.5225] [INSPIRE].

[5] WMAP collaboration, G. Hinshaw et al., Nine-Year Wilkinson Microwave Anisotropy Probe (WMAP) Observations: Cosmological Parameter Results, Astrophys. J. Suppl. 208 (2013) 19 [arXiv: 1212.5226] [INSPIRE].

[6] Planck collaboration, P.A.R. Ade et al., Planck 2013 results. I. Overview of products and scientific results, Astron. Astrophys. 571 (2014) A1 [arXiv:1303.5062] [INSPIRE].

[7] Planck collaboration, P.A.R. Ade et al., Planck 2013 results. XXII. Constraints on inflation, Astron. Astrophys. 571 (2014) A22 [arXiv:1303.5082] [INSPIRE].

[8] Supernova Search Team collaboration, B.P. Schmidt et al., The High Z supernova search: Measuring cosmic deceleration and global curvature of the universe using type-IA supernovae, Astrophys. J. 507 (1998) 46 [astro-ph/9805200] [INSPIRE].

[9] Supernova Search Team collaboration, A.G. Riess et al., Observational evidence from supernovae for an accelerating universe and a cosmological constant, Astron. J. 116 (1998) 1009 [astro-ph/9805201] [INSPIRE].

[10] Supernova Cosmology Project collaboration, S. Perlmutter et al., Measurements of Omega and Lambda from 42 high redshift supernovae, Astrophys. J. 517 (1999) 565 [astro-ph/9812133] [INSPIRE].

[11] G.W. Horndeski, Second-order scalar-tensor field equations in a four-dimensional space, Int. J. Theor. Phys. 10 (1974) 363 [inSPIRE].

[12] T. Kobayashi, M. Yamaguchi and J. Yokoyama, Generalized G-inflation: Inflation with the most general second-order field equations, Prog. Theor. Phys. 126 (2011) 511 [arXiv:1105.5723] [INSPIRE].

[13] C. Deffayet, X. Gao, D.A. Steer and G. Zahariade, From k-essence to generalised Galileons, Phys. Rev. D 84 (2011) 064039 [arXiv:1103.3260] [InSPIRE].

[14] G.W. Horndeski, Conservation of Charge and the Einstein-Maxwell Field Equations, J. Math. Phys. 17 (1976) 1980 [INSPIRE]. 
[15] C. Deffayet, A.E. Gümrükçüoğlu, S. Mukohyama and Y. Wang, A no-go theorem for generalized vector Galileons on flat spacetime, JHEP 04 (2014) 082 [arXiv:1312.6690] [INSPIRE].

[16] J. Gleyzes, D. Langlois, F. Piazza and F. Vernizzi, Healthy theories beyond Horndeski, Phys. Rev. Lett. 114 (2015) 211101 [arXiv:1404.6495] [INSPIRE].

[17] X. Gao, Unifying framework for scalar-tensor theories of gravity, Phys. Rev. D 90 (2014) 081501 [arXiv: 1406. 0822] [inSPIRE].

[18] M. Zumalacárregui and J. García-Bellido, Transforming gravity: from derivative couplings to matter to second-order scalar-tensor theories beyond the Horndeski Lagrangian, Phys. Rev. D 89 (2014) 064046 [arXiv:1308.4685] [INSPIRE].

[19] A. Padilla, P.M. Saffin and S.-Y. Zhou, Bi-galileon theory I: Motivation and formulation, JHEP 12 (2010) 031 [arXiv: 1007.5424] [INSPIRE].

[20] A. Padilla, P.M. Saffin and S.-Y. Zhou, Bi-galileon theory II: Phenomenology, JHEP 01 (2011) 099 [arXiv:1008.3312] [INSPIRE].

[21] K. Hinterbichler, M. Trodden and D. Wesley, Multi-field galileons and higher co-dimension branes, Phys. Rev. D 82 (2010) 124018 [arXiv:1008.1305] [INSPIRE].

[22] A. Padilla, P.M. Saffin and S.-Y. Zhou, Multi-galileons, solitons and Derrick's theorem, Phys. Rev. D 83 (2011) 045009 [arXiv: 1008.0745] [INSPIRE].

[23] A. Padilla and V. Sivanesan, Covariant multi-galileons and their generalisation, JHEP 04 (2013) 032 [arXiv:1210.4026] [INSPIRE].

[24] V. Sivanesan, Generalized multiple-scalar field theory in Minkowski space-time free of Ostrogradski ghosts, Phys. Rev. D 90 (2014) 104006 [arXiv:1307.8081] [INSPIRE].

[25] T. Kobayashi, N. Tanahashi and M. Yamaguchi, Multifield extension of G inflation, Phys. Rev. D 88 (2013) 083504 [arXiv: 1308.4798] [INSPIRE].

[26] S. Renaux-Petel, Orthogonal non-Gaussianities from Dirac- Born-Infeld Galileon inflation, Class. Quant. Grav. 28 (2011) 182001 [Erratum ibid. 28 (2011) 249601] [arXiv:1105.6366] [INSPIRE].

[27] S. Renaux-Petel, S. Mizuno and K. Koyama, Primordial fluctuations and non-Gaussianities from multifield DBI Galileon inflation, JCAP 11 (2011) 042 [arXiv:1108.0305] [INSPIRE].

[28] K. Koyama, G.W. Pettinari, S. Mizuno and C. Fidler, Orthogonal non-Gaussianity in DBI galileon: prospect for Planck polarization and post-Planck experiments, Class. Quant. Grav. 31 (2014) 125003 [arXiv:1303.2125] [INSPIRE].

[29] D. Langlois, S. Renaux-Petel, D.A. Steer and T. Tanaka, Primordial fluctuations and non-Gaussianities in multi-field DBI inflation, Phys. Rev. Lett. 101 (2008) 061301 [arXiv:0804.3139] [INSPIRE].

[30] D. Langlois, S. Renaux-Petel, D.A. Steer and T. Tanaka, Primordial perturbations and non-Gaussianities in DBI and general multi-field inflation, Phys. Rev. D 78 (2008) 063523 [arXiv: 0806.0336] [INSPIRE].

[31] H. Rund, Variational problems involving combined tensor fields, Abhandlungen aus dem Mathematischen Seminar der Universität Hamburg 29 (1966) 243.

[32] J.C. du Plessis, Tensorial concomitants and conservation laws, Tensor 20 (1969) 347. 